\title{
The congenial talking philosophers problem in computer networks
}

\author{
Yuh-Jzer Joung* \\ Department of Information Management, National Taiwan University, Taipei, Taiwan, Republic of China (e-mail: joung@ccms.ntu.edu.tw)
}

Received: August 2000 / Accepted: November 2001

Summary. Group mutual exclusion occurs naturally in situations where a resource can be shared by processes of the same group, but not by processes of different groups. For example, suppose data is stored in a CD-jukebox. Then when a disc is loaded for access, users that need data on the disc can concurrently access the disc, while users that need data on a different disc have to wait until the current disc is unloaded.

The design issues for group mutual exclusion have been modeled as the Congenial Talking Philosophers problem, and solutions for shared-memory models have been proposed [12, 14]. As in ordinary mutual exclusion and many other problems in distributed systems, however, techniques developed for shared memory do not necessary apply to message passing (and vice versa). So in this paper we investigate solutions for Congenial Talking Philosophers in computer networks where processes communicate by asynchronous message passing. We first present a solution that is a straightforward adaptation from Ricart and Agrawala's algorithm for ordinary mutual exclusion. Then we show that the simple modification suffers a severe performance degradation that could cause the system to behave as though only one process of a group can be in the critical section at a time. We then present a more efficient and highly concurrent distributed algorithm for the problem, the first such solution in computer networks.

Key words: Mutual exclusion - Group mutual exclusion Resource allocation - Distributed algorithms - Message passing

\section{Introduction}

Group mutual exclusion occurs naturally in situations where a resource can be shared by processes of the same group, but

This research was supported in part by the National Science Council, Taipei, Taiwan, under Grants NSC 86-2213-E-002-053 and NSC 872218-E-002-050, and by the 1998 Research Award of College of Management, National Taiwan University.

* Part of the research was done while the author was visiting Lab for Computer Science, Massachusetts Institute of Technology (19992000). not by processes of different groups. For example, suppose several users are working on a project which has some large data objects stored in a secondary memory such as a CDjukebox. When a user needs to access a data object, the data object is loaded from the secondary memory to a cache buffer. To increase performance, once a data object is loaded it will remain in the buffer until another data object is requested. So while a data object resides in the buffer, users that need to work on this data object are allowed to access the buffer (possibly a number of times), and users that need a different data object have to wait until no one is working on the data object in the buffer.

To capture the design issues of mutual exclusion intertwined with concurrency, we have presented a problem called Congenial Talking Philosophers (CTP) [12]. The problem concerns a set of $N$ philosophers $p_{1}, p_{2}, \ldots, p_{N}$ which spend their time thinking alone and talking in fora. The philosophers may like to hold $m$ different fora $\mathrm{X}_{1}, \ldots, \mathrm{X}_{m}$ but, due to the capacity of the meeting room, only one forum can be held at a time. However, more than one philosopher can be in a forum simultaneously. Initially, all philosophers are thinking. From time to time, when a philosopher is tired of thinking, it enters a waiting state where it wishes to attend a forum of its choice. A philosopher can choose only one forum at a time, but it may choose different fora at different waiting states. Given that there is only one meeting room - the shared resource, a philosopher attempting to enter the meeting room to attend a forum can succeed only if the meeting room is empty (and in this case the philosopher starts the forum), or some philosopher interested in the forum is already in the meeting room (and in this case the philosopher joins the ongoing forum). We assume that when a philosopher attends a forum, it spends an unpredictable but finite amount of time in the forum. After a philosopher leaves a forum (that is, exits the meeting room), it returns to thinking. ${ }^{1}$ We wish to design an algorithm for the philosophers satisfying the following requirements:

Mutual Exclusion: if some philosopher is in a forum, then no other philosopher can be in a different forum simultaneously.

\footnotetext{
1 Throughout the paper, "in a forum" is used synonymously with "in the meeting room." So, "to attend/leave a forum" is synonymous with "to enter/exit the meeting room."
} 
Lockout Freedom: a philosopher attempting to attend a forum will eventually succeed.

Concurrent Occupancy: if some philosopher $p$ has requested a forum $X$ and no philosopher is currently attending or requesting a different forum, then $p$ can attend $\mathrm{X}$ without waiting for other processes to leave the forum (see Appendix A.)

CTP is more general than the ordinary mutual exclusion problem and the Readers and Writers problem [5]. For mutual exclusion, we can dedicate one forum to each philosopher so that only one process can be in the critical section at a time. For the Readers and Writers problem, we can employ a READ operation (forum) for all processes (philosophers) in the system, and a unique WRITE operation for each individual process. A process attempting to read the shared object then requests the READ operation to access the object, while it requests its own WRITE operation when it wishes to update the object. Therefore, a shared object can be concurrently read by different processes, while writing alone must be mutually exclusive.

CTP also bears some similarity to the $k$-exclusion problem $[7,1]$ in that both allow multiple processes to attend a critical section. However, $k$-exclusion imposes a bound on the number of processes that may attend the critical section simultaneously, but does not distinguish between processes that may attend concurrently.

We are interested in completely decentralized solutions for the problem. A "semi-distributed" solution can be easily derived, for example, by employing a "concierge" for each forum. A philosopher interested in a particular forum first issues a request to the concierge of the forum. The concierges then compete with one another in a mutually exclusive style to obtain a privilege for their philosophers to use the meeting room. The algorithm is "semi-distributed" because although the contention for the meeting room is resolved in a decentralized manner by the concierges, the decision as to when a set of philosophers interested in the same forum can enter the meeting room is determined in a centralized manner by the concierge of the forum. Furthermore, the "semi-distributed" solution must process each request for a forum in two stages (and thus increases synchronization delay): one between the requesting philosopher and the corresponding concierge, and the other among the concierges for mutual exclusion.

In this paper we focus on CTP in computer networks where philosophers communicate by reliable and FIFO asynchronous message passing. Solutions for shared-memory models are treated in $[12,14,2]$. While it is true that sharedmemory algorithms can be systematically converted to message passing (or the other way around, see, e.g., Ch. 17 of [16]), such a transformation is generally costly. For example, the transformation of the shared-memory algorithm presented in [12] may result in an asymmetric solution where some processes are designated to maintain the shared variables, usually in a centralized manner, and so the processes are often the bottleneck of the performance. The solution also requires many messages (more than $6(N-1)$ ) per entry to the critical section. (These phenomena also occur in the transformation of the algorithms in [14,2].) Thus, it is worth investigating solutions that directly take advantage of the underlying features of the execution model. For example, two processes that wish to establish a communication in an asynchronous message-passing system simply execute a pair of send and receive commands. The system implicitly imposes a causal ordering on the execution so that the receive command cannot be executed until the message has arrived. In contrast, a more sophisticated technique is required in a completely decentralized shared-memory model to ensure that two asynchronous processes engaged in a communication are properly synchronized so that the information provider will not overwrite the information before the other process has observed the content.

Indeed, as we shall see shortly in Sect. 3, a symmetric and completely decentralized solution satisfying mutual exclusion, lockout freedom, and concurrent occupancy can be easily devised by slightly modifying Ricart and Agrawala's mutual exclusion algorithm [22]. ${ }^{2}$ This is not the case we have experienced in the shared-memory model; the algorithm presented in [12] is somewhat complex and is not a straightforward adaptation from existing algorithms for mutual exclusion. Nevertheless, one is easily deceived by this simple algorithm: its behavior appeared to be fine from static analysis until we put it on simulation and learned that it is only slightly better than one imposing mutual exclusion on every entry to the critical section! Therefore, it is also interesting to see why such a simple modification does not work and how a more efficient algorithm can be devised.

The rest of the paper is organized as follows: Sect. 2 presents some metrics for evaluating solutions for CTP. In Sect. 3 we present the straightforward solution described above and analyze why the solution has surprisingly poor performance. Section 4 then presents a more concurrent solution. Conclusions and future work are offered in Sect. 5.

\section{Complexity measures}

Solutions for CTP can be evaluated by two parameters: messages and time. For messages, like the ordinary mutual exclusion problem, we are concerned with the number of messages the system generates per entry to the critical section - the meeting room. For the time parameter, we are concerned with how long a philosopher may wait before entering a forum. Instead of using physical time - which would be system dependent and hard to analyze, we use passages as the basic metric for evaluating time, as defined below:

Definition 2.1 A passage by $p_{i}$ through the meeting room is an interval $\left[t_{1}, t_{2}\right]$, where $t_{1}$ is the time $p_{i}$ enters the meeting room, and $t_{2}$ the time it exits the meeting room. The passage is initiated at $t_{1}$, and is completed at $t_{2}$. The passage is ongoing at any time in between $t_{1}$ and $t_{2}$. The attribute of the passage is $\left\langle p_{i}, \mathrm{X}\right\rangle$, where $\mathrm{X}$ is the forum $p_{i}$ is attending.

When no confusion is possible, we use intervals (denoted by square brackets $\left[t_{1}, t_{2}\right]$ ) and attributes (denoted by angle brackets $\left.\left\langle p_{i}, \mathbf{X}\right\rangle\right)$ interchangeably to represent passages. The

\footnotetext{
${ }^{2}$ It is interesting to note that Ricart and Agrawala's algorithm can also be straightforwardly extended to solve $k$-exclusion [19]. As commented earlier, $k$-exclusion and CTP differ in that in $k$-exclusion any two processes can be in the critical section simultaneously so long as no more than $k$ processes are in the critical section, while in CTP any number of processes can be in the critical section simultaneously so long as they all belong to the same group. Thus solutions to $k$ exclusion cannot be directly applied to CTP, and vice versa.
} 


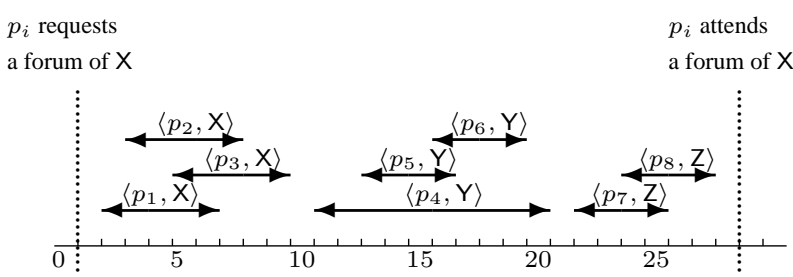

Fig. 1. A layout of passages

phrase "a passage through $\mathrm{X}$ by $p_{i}$ " refers to a passage with attribute $\left\langle p_{i}, \mathbf{X}\right\rangle$.

Definition 2.2 Let $S$ be a set of intervals. A subset $R$ of $S$ is a minimal cover of $S$ if for every $\alpha \in S$, every time instance in $\alpha$ is in some $\beta \in R$ (that is, $\forall\left[t_{1}, t_{2}\right] \in S, \forall t_{1} \leq t \leq t_{2}$ : $\left.\exists\left[t_{3}, t_{4}\right] \in R, t_{3} \leq t \leq t_{4}\right)$ and the size of $R$ is minimal. The dimension of $S$, denoted by $\operatorname{dim}(S)$, is the size of a minimal cover of $S$.

For example, Fig. 1 shows a scenario in which $p_{i}$ waits for eight passages before it attends a forum. The five passages $\left\langle p_{1}, \mathbf{X}\right\rangle,\left\langle p_{3}, \mathbf{X}\right\rangle,\left\langle p_{4}, \mathbf{Y}\right\rangle,\left\langle p_{7}, \mathbf{Z}\right\rangle$, and $\left\langle p_{8}, \mathbf{Z}\right\rangle$ constitute a minimal cover of the eight passages shown in the figure. Observe that passages may overlap. So when measuring the "time" $p_{i}$ waits in terms of passages, we cannot directly count all the passages it waits, but rather their dimension. So in this example only the above five passages suffice to account for $p_{i}$ 's wait.

The passage complexity is measured by $\operatorname{dim}(T)$, where $T$ is the maximal set of passages that may be initiated after a philosopher $p_{i}$ has made a request for a forum, and that must be completed before $p_{i}$ can enter the meeting room. Note that the definition of $T$ does not include those passages that are initiated after $p_{i}$ has made its request, but $p_{i}$ need not wait for them to be completed in order to enter the meeting room; that is, we do not count those passages that may be concurrently ongoing with $p_{i}$ 's.

In practice, a "context switch" occurs when a shared resource is "cleaned" for a new group of processes. For example, when a user requires a different data object in the shared objects environment described earlier in Sect. 1, the storage device has to unload the old object and then loads the new one. Depending on the applications, some context switches may be very time-consuming (such as disk loading and unloading). So in CTP a philosopher waiting for more passages through the same forum may in practice need less time than one waiting for fewer passages through different fora. So, in addition to the passage complexity, to measure the waiting time we also need to consider the number of "context switches" a philosopher may wait.

Definition 2.3 Let $U_{X}$ be a set of passages through $X$. Let $t_{s}=\min \left\{t \mid\left[t, t^{\prime}\right] \in U_{X}\right\}$, and $t_{f}=\max \left\{t^{\prime} \mid\left[t, t^{\prime}\right] \in\right.$ $\left.U_{X}\right\}$. Then, $U_{X}$ is a round of passages through $X$ (or simply a round of $X)$ if:

1. No passage other than those of $U_{X}$ is initiated in between $t_{s}$ and $t_{f} ;$ and

2. The last passage initiated before $t_{s}$ and the first passage initiated after $t_{f}$, if they exist, are for a different forum.
In other words, a round of $\mathrm{X}$ is a maximal set of consecutive passages through $\mathbf{X}$. If $U_{X}$ is a round of $\mathbf{X}$, then we say that the round is initiated at $t_{s}$, and completed at $t_{f}$.

The forum-switch (context-switch) complexity is measured by the maximum number of rounds of passages that may be initiated after a philosopher has made a request for $X$, but before a round of $\mathrm{X}$ is initiated in which $p_{i}$ makes a passage through $X$. For example, in Fig. $1 p_{i}$ waits for 3 rounds of passages for its request: a round of $X$, a round of $Y$, and a round of $Z$.

\section{A straightforward decentralized solution}

\subsection{The algorithm}

Recall that in Ricart and Agrawala's algorithm [22] for $n$ process mutual exclusion, a process requiring entry to the critical section multicasts a request message to every other process, and enters the critical section only when all other processes have replied to this request. To ensure mutual exclusion and lockout freedom, each process $p_{i}$ maintains a sequence number $S N_{i}$, initialized to 0 , that is to be updated according to Lamport's causality rules [15]. To issue a request, a process $p_{i}$ increases its $S N_{i}$ by 1 , and attaches the pair $\left\langle i, s n_{i}\right\rangle$ to the request, where $i$ is the unique identity of the process and $s n_{i}$ is the new value of $S N_{i} .\left\langle i, s n_{i}\right\rangle$ is used as the priority of the request.

Upon receiving a request with priority $\left\langle i, s n_{i}\right\rangle$, a process $p_{j}$ adjusts the value of its $S N_{j}$ to $\max \left(S N_{j}, s n_{i}\right)$, and uses the following rules to decide when to reply to the request:

1. $p_{j}$ replies immediately if it does not require entry to the critical section, or it requires entry to the critical section and the priority of its request is lower than $\left\langle i, s n_{i}\right\rangle$.

2 . The reply is delayed if $p_{j}$ also requires entry to the critical section and the priority of its request is higher than $\left\langle i, s n_{i}\right\rangle$. The reply is delayed until $p_{j}$ has exited the critical section.

The priority is ordered as follows: a priority $\left\langle i, s n_{i}\right\rangle$ is higher than $\left\langle j, s n_{j}\right\rangle$, denoted by $\left\langle i, s n_{i}\right\rangle \prec\left\langle j, s n_{j}\right\rangle$, if and only if $s n_{i}<s n_{j}$, or $s n_{i}=s n_{j}$ and $i<j$. Also, $\left\langle i, s n_{i}\right\rangle \preceq\left\langle j, s n_{j}\right\rangle$ if and only if $\left\langle i, s n_{i}\right\rangle \prec\left\langle j, s n_{j}\right\rangle$ or $\left\langle i, s n_{i}\right\rangle=\left\langle j, s n_{j}\right\rangle$.

Ricart and Agrawala's algorithm can be straightforwardly modified for CTP as follows: A philosopher wishing to attend a forum $X$ multicasts a request to every other philosopher, and enters the meeting room when all philosophers have replied to its request. A request message is of the form $\operatorname{Req}\left(\left\langle i, s n_{i}\right\rangle, \mathrm{X}\right)$, which additionally bears the name of the forum the philosopher wishes to attend. Also, a reply message (called an $a c$ knowledgment) is of the form $A c k(j)$, where $j$ is the identity of the replying philosopher.

Upon receipt of the request, a philosopher $p_{j}$ uses the following rules to decide when to reply to the request:

1. $p_{j}$ replies immediately if it is not interested in a different forum, or it is interested in a different forum and the priority of its request is lower than that of $p_{i}$ 's request. (A philosopher is interested in a forum if it has issued a request for the forum, and either it is still waiting to attend the forum, or it is already in the forum.)

2. The reply is delayed if $p_{j}$ is interested in a different forum and the priority of its request is higher than that of $p_{i}$ 's request. 
A.1 $*[$ wish to attend a forum of $\mathrm{X} \longrightarrow$

A.2 state $:=$ waiting;

A.3 $S N_{i}:=S N_{i}+1$;

A.4 multicast $\operatorname{Req}\left(\left\langle i, S N_{i}\right\rangle, \mathrm{X}\right)$ to every other philosopher; /* issue a request */

A.5 target $:=\mathrm{X}$;

A.6 priority $:=\left\langle i, S N_{i}\right\rangle ; / *$ store the priority */

B.1 $\square$ receive $\operatorname{Req}(\langle j, s n\rangle, \mathrm{Y}) \longrightarrow$

B.2 $S N_{i}:=\max \left(S N_{i}, s n\right)$; / adjust sequence number */

B.3 if $\langle j, s n\rangle \prec$ priority $\bigvee$ target $=\mathrm{Y}$ then

B. 4

B.5

C.1

C. 2

C. 3

C. 4

C.5

D. 1

D.2

D.3

D. 4

D.5

D.6

D.7 ]

send $A c k(i)$ to $p_{j} ; / *$ reply to the request immediately */

else requests_set $:=$ requests_set $\cup\{\operatorname{Req}(\langle j, s n\rangle, \mathrm{Y})\} ; / *$ defer the reply */

receive $A c k(j) \longrightarrow$

acks_set $:=a c k s \_s e t \cup\{A c k(j)\}$;

if $\mid$ acks_set $\mid=N-1$ then $\{/ *$ received replies from every other philosopher */ state $:=$ talking; $/ *$ enter the meeting room to attend a forum $* /$ acks_set $:=\emptyset ;\}$

exit a forum of target $\longrightarrow$

state $:=$ thinking;

target $:=\perp$;

priority $:=\langle i, \infty\rangle ; / *$ reset priority to a minimal value $* /$

for each $\operatorname{Req}(\langle j, s n\rangle, \mathrm{Y}) \in$ requests_set do send $A c k(i)$ to $p_{j}$;

requests_set $:=\emptyset$;

Variables:

- state: the state of $p_{i}$; see Fig. 3 for the state transition diagram. The initial state is thinking.

- $S N_{i}: p_{i}$ 's sequence number. It is initialized to 0 .

- priority: the priority of $p_{i}$ 's request. It is initialized to a minimal value $\langle i, \infty\rangle$.

- target: the forum $p_{i}$ wishes to attend, or $\perp$ otherwise. It is initialized to $\perp$.

- requests_set: the set of requests to which $p_{i}$ has not yet replied.

- acks_set: the set of replies to $p_{i}$ 's request.

Fig. 2. Algorithm RA1 executed by philosopher $p_{i}$

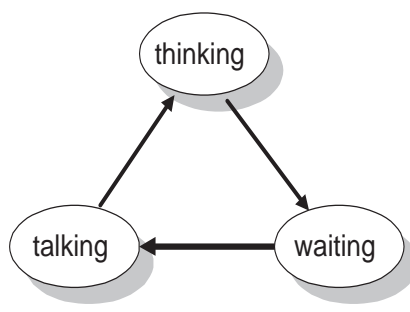

Fig. 3. State transition diagram of a philosopher

We refer to the modified algorithm as RA1. The complete code of RA1 is given in Fig. 2 as a CSP-like repetitive command [11], which is of the form

$$
*\left[g c_{1} \square g c_{2} \square \ldots \square g c_{k}\right]
$$

Each $g c_{i}$ is called a guarded command, which is of the form

$$
b \text {; receive } m s g \longrightarrow S
$$

where $b$ is a boolean condition called the boolean guard, and "receive msg" is called the message guard. Both the boolean guard and the message guard are optional. A guarded command can be executed only if it is enabled, i.e., its boolean guard evaluates to true and the specified message in the message guard has arrived. The execution receives the message and then the command $S$ is executed atomically. If there is more than one enabled guarded command, then one of them is chosen for execution, and the choice is nondeterministic. We do, however, require that a guarded command that is continuously enabled be executed eventually.

Note that like most algorithms for mutual exclusion (cf. [20]), we have assumed two threads of computation for each philosopher. One is responsible for the philosopher's normal activity in states thinking and talking, as well as the transition from thinking to waiting and from talking to thinking. The other is responsible for scheduling the transition from waiting to talking. It does so by sending and receiving messages to other philosophers, and by making the necessary assignments to local variables. A solution to the (group) mutual exclusion problem then specifies the behavior of the second thread.

Note further that in the algorithm, when $p_{i}$ issues a request $\operatorname{Req}(\langle i, s n\rangle, \mathrm{X})$, we let $p_{i}$ keep the priority $\langle i, s n\rangle$ in variable priority until it has left $\mathrm{X}$. The priority is reset to a minimal value $\langle i, \infty\rangle$ when $p_{i}$ is in state thinking. As such, a philosopher now possesses a priority all the time, and so the rules for a philosopher to decide when to reply to a request can be simplified as that shown in lines B.3-B.5.

\subsection{Analysis of RA1}

In this section we prove the correctness of RA1 and analyze its complexity. 


\section{Theorem 3.1 RAl guarantees mutual exclusion.}

Proof. Assume that $p_{i}$ and $p_{j}$ both have issued a request $\operatorname{Req}\left(\left\langle i, s n_{i}\right\rangle, \mathrm{X}\right)$ and $\operatorname{Req}\left(\left\langle j, s n_{j}\right\rangle, \mathrm{Y}\right)$, respectively. Without loss of generality, assume that $p_{i}$ 's priority is higher. By the way sequence numbers are maintained, $p_{j}$ 's request must be received by $p_{i}$ after $p_{i}$ has issued its request. When $p_{j}$ 's request arrives at $p_{i}$, if $\mathrm{X} \neq \mathrm{Y}$, then the reply to the request will be deferred by $p_{i}$ until $p_{i}$ has left $\mathrm{X}$. So the two philosophers cannot be in different fora simultaneously.

For the following theorem, we assume that a request for a forum ceases to exist once the requesting philosopher has completed a passage through the forum.

\section{Theorem 3.2 RAl guarantees lockout freedom.}

Proof. Observe that the sequence number $S N_{i}$ maintained by each $p_{i}$ is nondecreasing, and increases when the philosopher makes an entry to a forum. So after $p_{i}$ has issued a request $\operatorname{Req}(\langle i, s n\rangle, \mathrm{X})$, the number of requests with priorities higher than $\langle i, s n\rangle$ that could occur in the system is bounded. Moreover, because a philosopher can defer the reply to a request only if it has a request with priority higher than that of the requesting philosopher, and because a philosopher spends only finite time in a forum, the requests with priorities higher than $\langle i, s n\rangle$ will eventually cease to exist. Then all other philosophers will reply to $p_{i}$ 's request, thereby ensuring lockout freedom.

\section{Theorem 3.3 RAl allows concurrent occupancy.}

Proof. This follows from the fact that philosophers interested in the same forum do not delay one another's request. RA1.

The following theorem summarizes the static behavior of

Theorem 3.4 The message complexity, passage complexity, and forum-switch complexity of RAl are all $2(N-1)$.

Proof. For the message complexity, each entry to the meeting room requires $N-1$ requests and $N-1$ replies.

For the passage complexity, let $T$ be the set of passages that are initiated after $p_{i}$ has issued a request (say $\operatorname{Req}(\langle i, s n\rangle, \mathrm{X})$ ), and that must be completed before $p_{i}$ can enter the meeting room. We first argue that in between the time (call this $t_{1}$ ) $p_{i}$ issues a request and the time (call this $t_{2}$ ) it receives replies from all other philosophers, each $p_{j}$ can have at most two requests with priority higher than that of $p_{i}$ 's request. This is because messages over a communication link are delivered in FIFO order. So if $p_{i}$ receives a request by $p_{j}$ after $p_{i}$ has issued $\operatorname{Req}(\langle i, s n\rangle, \mathrm{X})$, then before $p_{j}$ issues a new request, it must have received $p_{i}$ 's reply to its previous request, and so it must have received $p_{i}$ 's request $\operatorname{Req}(\langle i, s n\rangle, \mathrm{X})$ sent before the reply. So $p_{j}$ 's new request must have priority lower than that of $p_{i}$ 's request. So in between $t_{1}$ and $t_{2}, p_{j}$ can have at most two requests with priority higher than that of $p_{i}$ 's request: one that $p_{i}$ receives before $t_{1}$, and the other that $p_{i}$ receives after $t_{1} .^{3}$

\footnotetext{
${ }^{3}$ The FIFO assumption can be lifted if acknowledgments are also "timestamped" by sequence numbers.
}

The above argument implies that $|T| \leq 2(N-1)$. It is easy to see that the passages in $T$ can be pairwise disjoint. So the passage complexity of RA1 is $2(N-1)$. The forumswitch complexity is also $2(N-1)$ because two consecutive passages in $T$ can be for different fora.

We now study how RA1 responds to contention. We first observe that RA1 adopts the following "entry policy":

\section{No philosopher can attend a forum if there is a higher priority request from a different philosopher waiting to enter a different forum.}

So the order of entries to the meeting room will entirely be based on request priorities. To analyze RA1, we consider a scenario in which $l \cdot k$ (out of the $N$ ) philosophers wish to attend a forum simultaneously. Assume that the philosophers are divided into $l$ groups, each of which consists of $k$ philosophers, such that philosophers in the same group wish to attend the same forum.

Suppose that the priorities of the philosophers' requests are ordered decreasingly as follows:

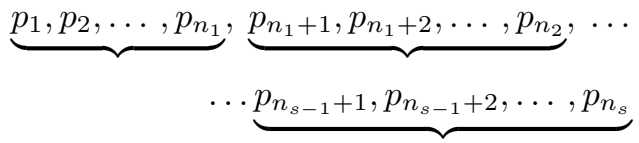

where philosophers $p_{n_{i-1}+1}, p_{n_{i-1}+2}, \ldots, p_{n_{i}}$ all wish to attend the same forum, and the forum is different from the forum targeted by $p_{n_{i}+1}, p_{n_{i}+2}, \ldots, p_{n_{i+1}}, 1 \leq i \leq s$ (and let $n_{0}=0$ ). By the algorithm, the $l \cdot k$ requests will result in $s$ rounds of passages (because the philosophers will enter the meeting room in the order of the priorities of their requests), where the $i$ th round consists of $n_{i}-n_{i-1}$ passages.

Consider the expected value of $s$ and $n_{i}-n_{i-1}$. Observe that there are $(l \cdot k)$ ! possible ways of ordering the $l \cdot k$ requests. Suppose that the $l \cdot k$ requests have equal probability to assume each ordering. Let $E(l, k)$ be the expected number of rounds of passages the requests may generate. In combinatorics, $E(l, k)$ is equivalent to the expected number of runs $l \cdot k$ balls, $k$ balls per color, may generate when they are randomly placed on a line, where a run is defined to be a maximal sequence of balls of the same color. By a combinatorial analysis [6], we have

$E(l, k)=l k-k+1$

Given that there are $l \cdot k$ requests, the expected number of passages per round is

$\frac{l k}{l k-k+1}<\frac{l k}{l k-k}=\frac{l}{l-1}$

Therefore, when $l$ increases, the expected number of passages per round tends to be one. So we can expect that when the number of fora $m$ that philosophers may compete for is large, RA1 behaves like a mutual exclusion algorithm where only one philosopher can be in the critical section at a time. Even when there are only two fora (i.e., $m=2$ ), at most two philosophers are expected to attend a forum concurrently, regardless of how many philosophers are in each group.

Note that the above analysis is based on the assumption that at any snapshot of the system, the order of priorities of existing requests to the meeting room is randomly distributed. This 
assumption in turn is based on the observation that after two philosophers $p_{i}$ and $p_{j}$ have attended a forum, the next time they make a request, either one of them can obtain a priority higher than the other, regardless of whose priority was higher in their previous requests. This is because if $p_{i}$ 's new request arrives at $p_{j}$ before $p_{j}$ makes its request, then $p_{j}$ 's priority will be lower than $p_{i}$ 's. Similarly, $p_{i}$ 's priority will be lower than $p_{j}$ 's if $p_{j}$ 's new request arrives at $p_{i}$ before $p_{i}$ makes its request. If both philosophers make their requests concurrently, then their priorities are determined by their sequence numbers $S N_{i}$ and $S N_{j}$ at the time they make the requests, with tie breakers being based on philosopher ids. For simplicity, in the analysis we have assumed an equal chance for either $p_{i}$ or $p_{j}$ to win the priority. As we shall see shortly, the results measured in our simulation are close to the above analysis.

\subsection{Simulation results}

In the simulation, we set up a system of $N$ philosophers and $m$ fora. The forum a philosopher may choose when it wishes to attend a forum is set up in two different ways: fixed and random. In the fixed forum choice, the $N$ philosophers are divided into $m$ disjoint groups. Each group is assigned a unique forum so that philosophers in the group always choose the forum to attend when they wish to attend a forum. In the random forum choice, a philosopher randomly chooses one of the $m$ fora to attend each time it wishes to attend a forum. To distinguish between these two settings, we put an extra star ' $*$ ' on the value of $m$ to indicate that the forum choice is fixed. Thus, when $m=N *$, the philosophers will use the meeting room in a mutually exclusive style.

The time a philosopher stays in state thinking and in state talking follows an exponential distribution with means $\mu_{\text {thinking }}$ and $\mu_{\text {talking }}$ respectively. The message transmission time also follows an exponential distribution with a mean $\mu_{\text {link_delay. }}$ In the simulation we fixed $\mu_{\text {talking }}$ to be $250 \mathrm{~ms}$, and $\mu_{\text {link_delay }}$ to be $2 \mathrm{~ms}$. The choice of these values came from the observations that CD-ROM access time ranges from $500 \mathrm{~ms}$ to several milliseconds (depending on the speed) ${ }^{4}$, and that message delays in LAN are typically in the order of several milliseconds. Note that we do not consider forum switch cost. If needed, the cost per request can be calculated by multiplying the time needed for a forum switch, and the average number of forum switches a request has to wait. The simulation program is written in Java. ${ }^{5}$.

In the first experiment we studied how RA1 reacts to contention. As can be seen, when $\mu_{\text {talking }}$ is fixed, the larger the value $\frac{\mu_{\text {talking }}}{\mu_{\text {thinking }}+\mu_{\text {talking }}}$, the more often a philosopher wishes to attend a forum, and so the higher the level of contention. So we used $\frac{\mu_{\text {talking }}}{\mu_{\text {thinking }}+\mu_{\text {talking }}}$ as a metric for contention. We varied $\mu_{\text {thinking }}$ from $9000 \mathrm{~ms}$ to $0 \mathrm{~ms}$ along the $x$-axis. We measured the following three values: (i) average round size, i.e., the average number of passages per round, (ii) average number of forum switches a request has to wait, and (iii) throughput ra-

\footnotetext{
${ }^{4}$ See PC Magazine CD-ROM Drives Performance Tests, http://www.zdnet.com/pcmag/features/cdrom/ cd-test.htm

${ }^{5}$ Some Java applets animating the algorithms for CTP can be found in http://joung.im.ntu.edu.tw/congenial/
}

tio $T / T_{0}$, where $T$ is the number of entries to the meeting room per second, and $T_{0}$ is the number of entries to the meeting room per second when the philosophers use the meeting room in a mutually exclusive style. For comparison, we set $m$ to the following values $2,3,5,10$, and 30 (for random forum choice), and $2 *, 3 *, 5 *, 10 *$, and $30 *$ (for fixed forum choice). The results are shown in the six charts of Fig. 4. Each data point in the charts is measured by letting each philosopher make approximately 500 requests.

The two charts on the top show the results measured for average round size. We can see that when the forum choice is random (left chart), the average round size is close to $\frac{m}{m-1}$ as analyzed in the previous section (see Equation (2)), regardless of the level of contention. When the forum choice is fixed, the average round size is close to $\frac{m}{m-1}$ when the contention is low, and it decreases slightly when the level of contention increases. Intuitively, when the contention is high, more philosophers wish to attend the same forum simultaneously. So if an algorithm handles concurrency well, then the average round size should increase. From the experimental results we see that RA1 has poor concurrency performance. Likewise, we can expect that the number of forum switches in RA1 will approach to $N-N / m+1$ when the level of contention increases (see Equation (1) in Sect. 3.2). This is confirmed by the experimental results shown in the two middle charts. Similarly, the throughput ratio measured in the two bottom charts show that little concurrency is offered by RA1.

In the second experiment we studied the performance of RA1 with respect to the number of fora $m$. Because not all values of $m$ can divide $N$, we use random forum choice in the experiment. Again, we measured the following: (i) average round size, (ii) average number of forum switches, and (iii) throughput ratio. The results are shown on the three charts of Fig. 5. Each chart shows five curves for each of the following values of $\mu_{\text {thinking }}$ (in millisecond): $0,250,500,1000$, and 4000 , corresponding to the following levels of contention: $100 \%, 50 \%, 33.3 \%, 20 \%$, and $5.9 \%$. From the results we see that when $m$ increases, RA1 performs like a mutual exclusion algorithm regardless of the level of contention.

\section{A highly concurrent solution}

As analyzed above, the poor concurrency of RA1 is due to the entry policy that no philosopher can attend a forum if there is a higher priority request from a different philosopher waiting to enter a different forum. As a result, if two philosophers $p_{i}$ and $p_{j}$ are interested in the same forum, but a third philosopher $p_{k}$ interested in a different forum has obtained a priority in between $p_{i}$ 's and $p_{j}$ 's, then $p_{i}$ and $p_{j}$ cannot attend a forum concurrently. Clearly, concurrency can be increased if $p_{j}$ can join $p_{i}$ 's forum. To do so, we can weaken the entry policy to the following:

$$
\begin{aligned}
& \text { No philosopher } p_{j} \text { can attend a forum } \mathrm{X} \text { if there is a } \\
& \text { higher priority request from some } p_{k} \text { waiting to enter } \\
& \text { a different forum, unless there is another } p_{i} \text { already in } \\
& \mathrm{X} \text { such that } p_{i} \text { 's request has priority higher than } p_{k} \text { 's } \\
& \text { request. }
\end{aligned}
$$

In the following we present an algorithm to implement the policy. 

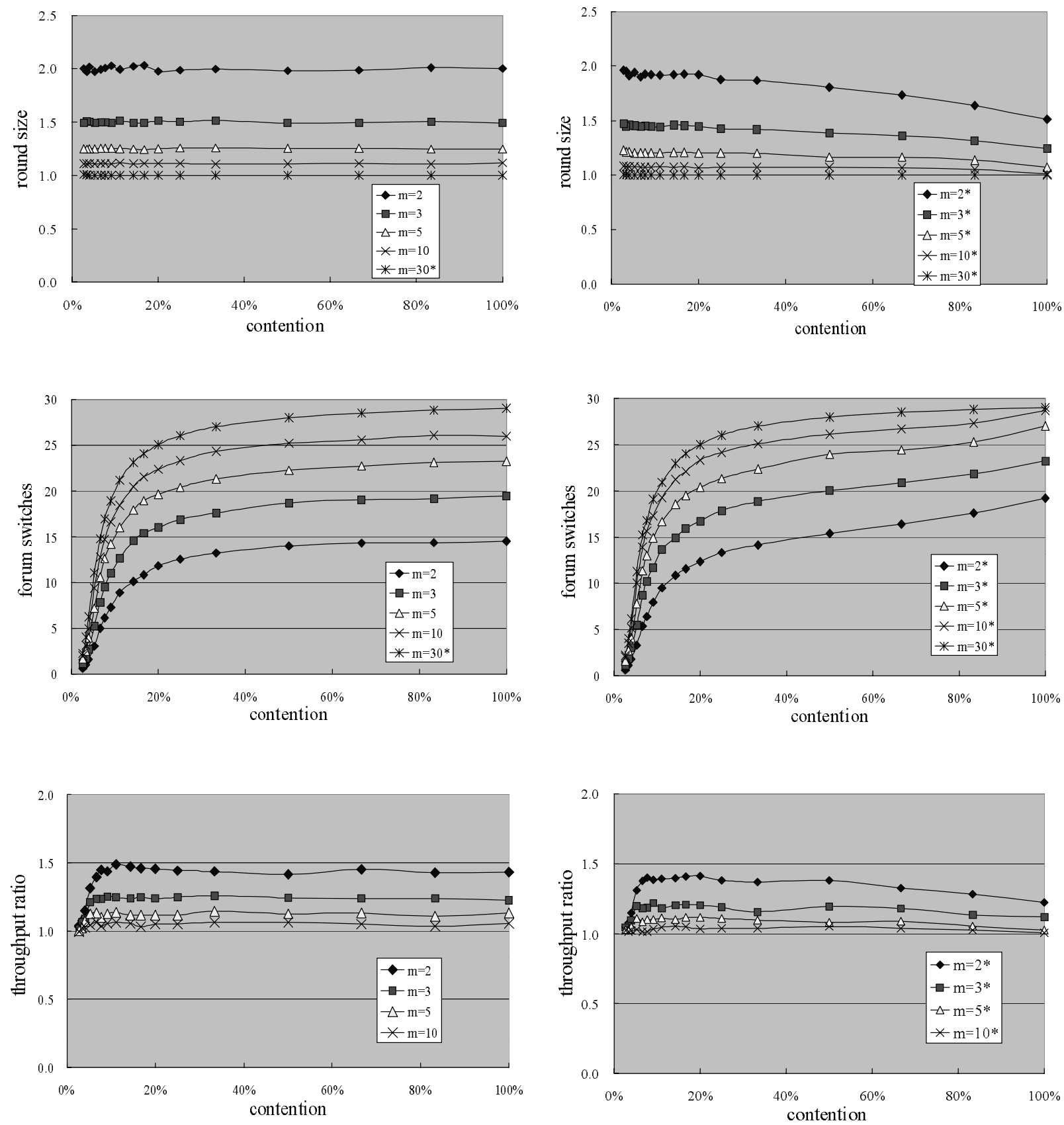

Fig. 4. Performance of RA1 with respect to contention. The left three charts are for random forum choice, where as the right three charts are for fixed forum choice

\subsection{The algorithm}

By the new entry policy, we can distinguish two types of philosophers in a forum: ${ }^{6}$

- Philosophers that attend the forum because there is no other philosopher with a higher priority such that the philosopher is interested in a different forum. We call these philosophers captains.

- Philosophers that attend the forum because there is a captain in the forum. We call these philosophers successors.

${ }^{6}$ Note that the definitions of the two types are not disjoint: a philosopher that can potentially become a captain in a forum may become a successor.
In RA1, every philosopher $p_{i}$ in a forum can be considered as a captain. It enters the forum by receiving acknowledgments to its request from all other philosophers in the system. To implement the concept of successors, we can modify RA1 to let $p_{i}$ in a forum capture other philosophers requesting the same forum to enter the forum. Capturing can be done by leting $p_{i}$ reply to their requests with a Start message that tells them to enter the meeting room directly without waiting for acknowledgments from other philosophers. For now, we do not allow successors to capture. Section 4.4 considers extensions of the algorithm that allow successors to capture as well.

In the above scenario, when $p_{i}$ replies a Start to $p_{j}$, another $p_{k}$ with priority lower than $p_{i}$ may have also issued a request 

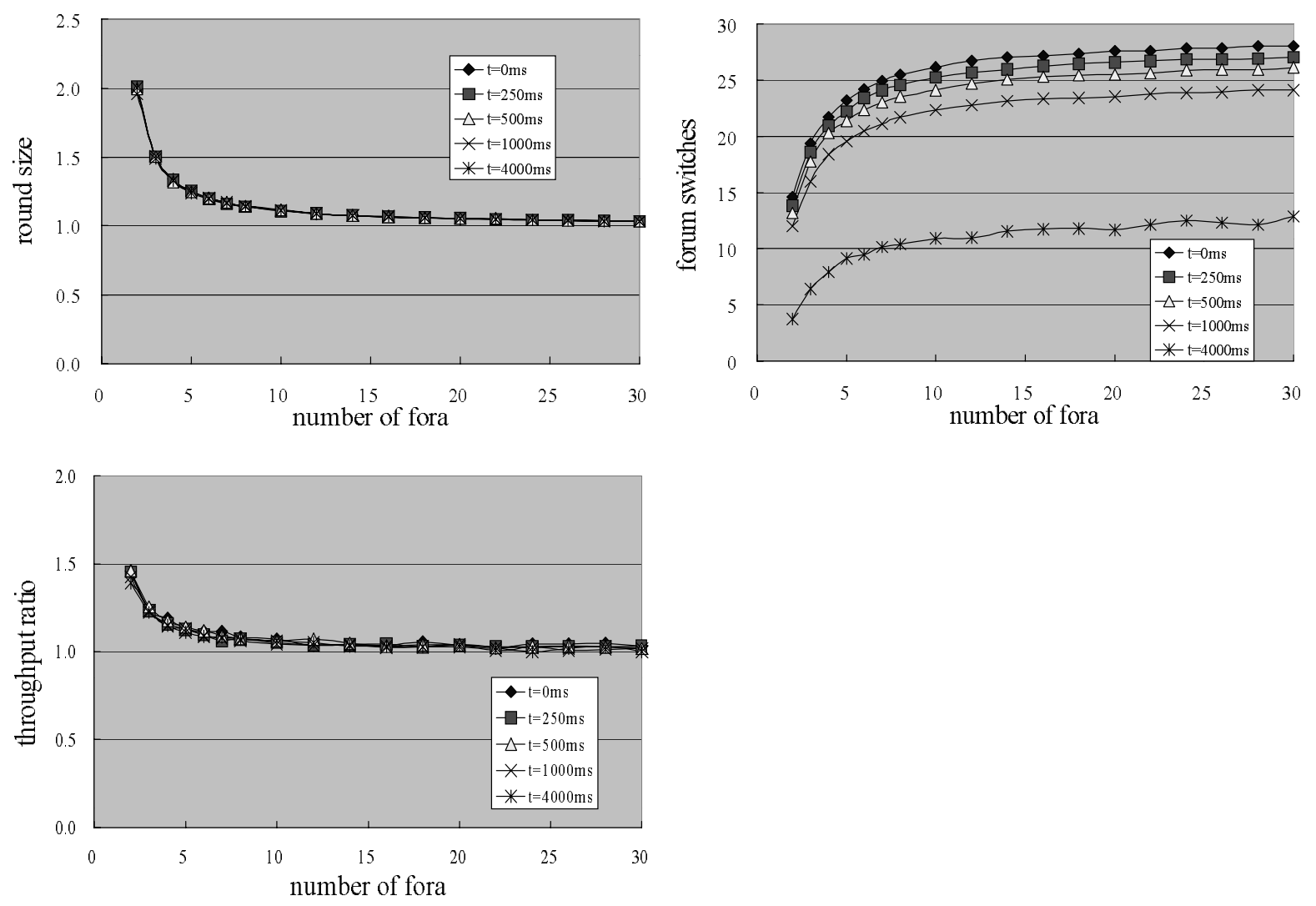

Fig. 5. Performance of RA1 with respect to the number of fora $m$. Time $t$ in each chart shows the value of $\mu_{\text {thinking }}$

for a different forum, and may have already obtained $p_{j}$ 's acknowledgment, waiting for only $p_{i}$ 's reply. So $p_{j}$ 's entry to the meeting room must be known to $p_{k}$. To do so, when $p_{i}$ leaves the forum, in the reply to $p_{k}$ 's request, $p_{i}$ informs $p_{k}$ that it has captured $p_{j}$ to attend a forum. $p_{k}$ then uses this information to decide if it has an "up-to-date" reply from $p_{j}$. If so, then $p_{k}$ is sure that $p_{j}$ has also exited the forum and so can enter the meeting room; otherwise, $p_{k}$ must wait for a new reply from $p_{j}$. Clearly, $p_{j}$ must send such a reply on exiting the meeting room.

From the above discussion we see that sequence numbers alone do not convey enough information for philosophers to decide whether a reply to a request is out-of-date. So in the new algorithm, referred to as RA2, each philosopher $p_{i}$ maintains a vector sequence number $V S N_{i}$ [18]. $V S N_{i}$ is a vector of natural numbers of length $N$ and is initialized to contain all zeros. The value $V S N_{i}[j]$ represents a count of requests by $p_{j}$ that are known at $p_{i}$, either because they originate there (when $j=i$ ) or because their existence is known through message passing. Let $\mathcal{V} \mathcal{S}$ denote the set of vectors of natural numbers of length $N$, and $\mathcal{N}$ denote the set of natural numbers. The binary relation ' $\prec$ ' on $\mathcal{N} \times \mathcal{V} \mathcal{S N}$ is defined as follows:

$$
\begin{aligned}
\langle j, u\rangle \prec\langle k, v\rangle & \text { iff } \sum_{l} v[l]>\sum_{l} u[l] \\
\text { or }\left(\sum_{l} v[l]\right. & \left.=\sum_{l} u[l] \wedge k>j\right)
\end{aligned}
$$

It can be seen that the binary relation ' $\prec$ ' on $\mathcal{N} \times \mathcal{V} \mathcal{S N}$ is antisymmetric and transitive; and for any two pairs $\langle j, u\rangle$ and $\langle k, v\rangle$ such that $j \neq k$, either $\langle j, u\rangle \prec\langle k, v\rangle$ or $\langle k, v\rangle \prec\langle j, u\rangle$.

Furthermore, $p_{i}$ also maintains a vector $V F_{i}$ of natural numbers of length $N$, where $V F_{i}[j]$, initialized to 0 , represents a count of entries to the meeting room that are made by $p_{j}$ and that are known at $p_{i}$.

The vectors are used as follows: When a philosopher $p_{i}$ wishes to attend a forum $\mathrm{X}$, it increments $V S N_{i}[i]$ by 1 and, like RA1, $p_{i}$ multicasts a request message $\operatorname{Req}\left(\left\langle i, v s n_{i}\right\rangle, \mathrm{X}\right)$ to every other philosopher, where $v s n_{i}$ is the new value of $V S N_{i}$. The value $\left\langle i, v s n_{i}\right\rangle$ is the priority of the request. A priority $\langle j, u\rangle$ is higher than $\langle k, v\rangle$ if and only if $\langle j, u\rangle \prec\langle k, v\rangle$. Unlike RA1, however, $p_{i}$ enters the meeting room either because every philosopher has replied with an "up-to-date" acknowledgment $A c k$, or because some philosopher has replied with a message Start. In the former case, $p_{i}$ enters the meeting room as a captain, (and acts as a captain in the meeting room), while in the latter case $p_{i}$ enters the meeting room as a successor. Note that many captains may co-exist in a forum.

Upon receiving a request $\operatorname{Req}\left(\left\langle i, v s n_{i}\right\rangle, \mathrm{X}\right), p_{j}$ updates $V S N_{j}$ to merge $\left(V S N_{j}, v s n_{i}\right)$, where function merge $(u, v)$ is defined as follows:

$$
\operatorname{merge}(u, v)[k]=\max (u[k], v[k]), \quad 1 \leq k \leq N
$$

It can be seen that if a request with priority $\langle j, u\rangle$ "happens before" (in Lamport's causality relation [15]) a request with priority $\langle k, v\rangle$, then $\sum_{l} u[l]<\sum_{l} v[l]$, and so $\langle j, u\rangle \prec\langle k, v\rangle$.

The rules to decide when and how to reply to the request are as follows:

1. $p_{j}$ replies with an acknowledgment $A c k\left(j, v s n_{i}[i], v f_{j}\right)$ (where $v f_{j}$ is the current value of $V F_{j}$ ) immediately if either (1) it is also interested in X but is not currently acting as a captain in the meeting room, or (2) it is not interested 
in $\mathrm{X}$, is not in the meeting room, and has a priority lower than $\left\langle i, v s n_{i}\right\rangle$. (Like RA1, we let $p_{j}$ possess a priority all the time. The priority is set to $\left\langle j, v s n_{j}\right\rangle$ when $p_{j}$ issues a request $\operatorname{Req}\left(\left\langle j, v s n_{j}\right\rangle, \mathrm{Y}\right)$, and is reset to a minimal value $\langle j,[\infty, \ldots, \infty]\rangle$ when $p_{i}$ exits $\mathrm{Y}$.)

2. $p_{j}$ replies with a message $\operatorname{Start}\left(\left\langle j, v s n_{j}\right\rangle, v s n_{i}[i]\right)$ if it is in forum $\mathrm{X}$ and is acting as a captain in the meeting room. Note that the reply bears $p_{j}$ 's current priority $\left\langle j, v s n_{j}\right\rangle$.

3. Otherwise, $p_{j}$ must be interested in a different forum, and either $p_{j}$ is in the meeting room, or its priority is higher than $\left\langle i, v s n_{i}\right\rangle$. In this case, $p_{j}$ delays the reply until it has exited the meeting room, and then replies to $p_{i}$ 's request with an acknowledgment.

Notice that an acknowledgment $A c k\left(j, v s n_{i}[i], v f_{j}\right)$ in the algorithm additionally carries two values: $v s n_{i}[i]-p_{i}$ 's sequence number in its request, and $v f_{j}-p_{j}$ 's knowledge (at the time the acknowledgment is sent) of the counts of entries to the meeting room made by each philosopher. The value $v s n_{i}[i]$ is used by $p_{i}$ to determine whether the acknowledgment is to its current request, or to a previous one. In the latter case the acknowledgment is out-of-date and must be discarded. A philosopher may receive an out-of-date acknowledgment because it can enter the meeting room (as a successor) before it receives all acknowledgments to its request.

The value of $v f_{j}$ is for $p_{i}$ to update its $V F_{i}: p_{i}$ updates $V F_{i}$ to merge $\left(V F_{i}, v f_{j}\right)$ on receipt of $A c k\left(j, v s n_{i}[i], v f_{j}\right)$. The new value then is used by $p_{i}$ to check if every acknowledgment $\operatorname{Ack}\left(k, v s n_{i}[i], v f_{k}\right)$ it possesses remains up-to-date. In the algorithm, $V F_{i}[k]$ must be no greater than $v f_{k}[k]$ in order for $A c k\left(k, v s n_{i}[i], v f_{k}\right)$ to remain up-to-date. The intuition is: $V F_{k}[k]$ must always have a correct count of the number of entries to the meeting room $p_{k}$ has made. No $p_{j}$ can have its $V F_{j}[k]$ greater than $V F_{k}[k]$ unless some $p_{l}$ has captured $p_{k}$, and has increased $V F_{l}[k]$ to $V F_{k}[k]+1$ before $p_{k}$ receives $p_{l}$ 's start message. So if $V F_{i}[k]$ is greater than $v f_{k}[k]$ carried by $p_{k}$ 's acknowledgment $A c k\left(k, v s n_{i}[i], v f_{k}\right)$, then $p_{i}$ knows that $p_{k}$ has been captured to attend a forum after replying the acknowledgment to $p_{i}$. So $p_{i}$ must wait for a new acknowledgment from $p_{k}$ to make sure that $p_{k}$ has exited the forum.

The complete code of the algorithm is given in Fig. 6 . Again, the algorithm is presented as a CSP-like repetitive command consisting of five guarded commands $\mathrm{A}, \mathrm{B}, \mathrm{C}, \mathrm{D}$, and E.

Messages are of the following three types:

- $\operatorname{Req}\left(\left\langle i, v s n_{i}\right\rangle, \mathrm{X}\right)$ : a request for forum $\mathrm{X}$ by $p_{i}$, where $\langle i$, $\left.v s n_{i}\right\rangle$ is the priority of the request.

- $\operatorname{Ack}\left(i, s n, v f_{i}\right)$ : an acknowledgment by $p_{i}$ to $p_{j}$ 's $s n$th request. $v f_{i}$ is the value of $V F_{i}$ at the time the acknowledgment is sent.

- $\operatorname{Start}\left(\left\langle i, v s n_{i}\right\rangle, s n\right)$ : a reply by $p_{i}$ to $p_{j}$ 's $s n$th request; the reply informs $p_{j}$ that it can directly enter the meeting room. $\left\langle i, v s n_{i}\right\rangle$ is the priority of $p_{i}$ at the time the message is sent.

Some detailed comments on the algorithm are provided below. First, consider guarded command B that processes request messages. After $p_{i}$ has replied with an acknowledgment to $\operatorname{Req}\left(\left\langle j, v s n_{j}\right\rangle, \mathrm{Y}\right), p_{i}$ may need to send another reply to the request later on. This happens when (1) $p_{i}$ subsequently enters the meeting room as a captain and wishes to capture $p_{j}$ to join the forum, or (2) $p_{i}$ is captured to attend the meeting room and needs to send a new acknowledgment to $p_{j}$ after it has exited the meeting room. Therefore, a request message may need to be saved for another reply. In the algorithm, request messages are kept in requests_set. Obviously, only the most recent request per philosopher needs to be saved; see lines B.3-4. Lines B.7-13 implement the three rules described earlier regarding how request messages are processed. Each new request is kept in requests_set (line B.6) until $p_{i}$ determines that it is out-of-date; see the comment below.

Acknowledgments are processed by guarded command C. $p_{i}$ enters the meeting room as a captain only when its request has been acknowledged by every other philosopher (line C.10). Then, for each request for $\mathrm{X}$ in requests_set, $p_{i}$ sends a Start message to capture the requesting philosopher to join the forum (lines C.16-19). The requests in requests_set bearing a priority higher than that of $p_{i}$ 's request can now be deleted (lines C.20-21) because the requesting philosophers must have already entered the meeting room for their requests, for otherwise they will not acknowledge $p_{i}$ 's request. If $p_{i}$ instead enters the meeting room as a successor (line D.3), then it removes the captain's request and the requests in requests_set that bear a priority higher than that of the captain's request (lines D.7-8). The captain's priority carried by a start message is used to determine which requests have ceased to exist.

On exiting the meeting room, $p_{i}$ must reply to the requests that are held in requests_set. Not every request needs to be replied to, though. For example, if the value of $v s n_{j}[j]$ in a request $\operatorname{Req}\left(\left\langle j, v s n_{j}\right\rangle, \mathrm{Y}\right)$ is less than or equal to $V F_{i}[j]$, then, obviously, the request has been granted and so no reply is needed. Moreover, if $p_{i}$ acknowledges a request $\operatorname{Req}\left(\left\langle j, v s n_{j}\right\rangle, \mathrm{Y}\right)$ while it is in $\mathrm{Y}$, then the acknowledgment must carry the most up-to-date count of entries $p_{i}$ has made to the meeting room. So $p_{i}$ need not send a new acknowledgment upon exiting the forum. The set friend_requests is used to store such requests (lines B.14-16 and E.3). Lines E.2-6 determine which request needs to be replied and which needs to be removed.

Note that after $p_{i}$ has replied to a request (say, by $p_{j}$ ) of the same interest (say, forum $\mathrm{Y}$ ), $p_{i}$ may still need to keep the request in requests_set. This is because there is no guarantee that $p_{j}$ 's request will be granted before or concurrently with $p_{i}$ 's request regardless of whose priority is higher. For example, suppose $p_{i}$ is captured by $p_{k}$ to attend $Y$. Suppose further that $p_{j}$ 's request arrives at $p_{k}$ after $p_{k}$ has left $Y$ (so that $p_{k}$ is unable to capture $p_{j}$ in time), and $p_{j}$ 's request arrives at $p_{i}$ before $p_{k}$ has captured $p_{i}$. Then, when $p_{i}$ acknowledges $p_{j}$ 's request, $p_{j}$ 's knowledge of $V F_{i}[i]$ is one less than the count it learns from $p_{k}$ 's acknowledgment later on. So $p_{i}$ must send a new acknowledgment to $p_{j}$ upon exiting $\mathrm{Y}$. Moreover, after $p_{i}$ has sent the new acknowledgment, $p_{i}$ may request another forum and then be captured again to enter the meeting room before $p_{j}$ is allowed to enter the meeting room. So $p_{i}$ 's second acknowledgment again becomes out-of-date and a new one must be issued. In the worst case, $p_{i}$ needs to keep $p_{j}$ 's request until it learns that the request has ceased to exist.

Finally, suppose $p_{k}$ captures $p_{j}$, and let $\operatorname{Req}\left(\left\langle j, v s n_{j}\right\rangle, \mathrm{X}\right)$ be $p_{j}$ 's request. Because $p_{k}$ sets its $V F_{k}[j]$ to $v s n_{j}[j]$ upon capturing $p_{j}$, until $p_{j}$ receives $p_{k}$ 's start message $V F_{k}[j]$ is greater than $V F_{j}[j]$ (whose value is $v s n_{j}[j]-1$ when $p_{j}$ issues its request). In the meantime, another $p_{i}$ may learn 
A.1 $*$ [wish to attend a forum of $\mathrm{X} \longrightarrow$

A. 2

A. 3

A. 4

A. 5

A. 6

B.1

B. 2

B. 3

B. 4

B. 5

B. 6

B. 7

B.8

B. 9

B. 10

B. 11

B. 12

B. 13

B. 14

B. 15

B. 16

B. 17

C. 1

C. 2

C. 3

C. 4

C. 5

C. 6

C.7

C. 8

C. 9

C. 10

C. 11

C. 12

C. 13

C. 14

C. 15

C. 16

C. 17

C. 18

C. 19

C. 20

C. 21

C. 22

C. 23

D. 1

D. 2

D. 3

D. 4

D. 5

D. 6

D. 7

D. 8

D. 9
$V S N_{i}[i]:=\operatorname{VSN}_{i}[i]+1$;

target $:=\mathrm{X}$

priority $:=\left\langle i, V S N_{i}\right\rangle ;$ / $^{*}$ obtain a priority for its request */

state $:=$ waiting;

multicast $\operatorname{Req}\left(\left\langle i, V S N_{i}\right\rangle, \mathrm{X}\right)$ to every other philosopher; /* issue a request */

$\square$ receive $\operatorname{Req}\left(\left\langle j, v s n_{j}\right\rangle, \mathrm{Y}\right) \longrightarrow$

$V S N_{i}:=\operatorname{merge}\left(V S N_{i}, v s n_{j}\right) ; / *$ adjust $V S N_{i} * /$

if $\exists \operatorname{Req}\left(\left\langle j, v s n_{j}^{\prime}\right\rangle, \mathrm{Z}\right) \in$ requests_set then $/ *$ remove $p_{j}$ 's previous request */

requests_set $:=$ requests_set $-\left\{\operatorname{Req}\left(\left\langle j, v s n_{j}^{\prime}\right\rangle, \mathbf{Z}\right)\right\}$;

if $V F_{i}[j]<v s n_{j}[j]$ then \{

requests_set $:=$ requests_set $\cup\left\{\operatorname{Req}\left(\left\langle j, v s n_{j}\right\rangle, \mathrm{Y}\right)\right\}$;

if $($ target $=\mathrm{Y} \wedge \neg$ is_captain $) \bigvee\left(\right.$ target $\neq \mathrm{Y} \wedge$ state $\neq$ talking $\wedge\left\langle j, v s n_{j}\right\rangle \prec$ priority $)$ then send $A c k\left(i, v s n_{j}[j], V F_{i}\right)$ to $p_{j}$;

else if target $=\mathrm{Y} \wedge$ is_captain then \{

send $\operatorname{Start}\left(\right.$ priority, $\left.v s n_{j}[j]\right)$ to $p_{j} ; / *$ capture $p_{j} * /$

$I^{*} p_{i}$ is sure that $p_{j}$ will make its $v s n_{j}[j]$ th entry to the meeting room */

$V F_{i}[j]:=\max \left(V F_{i}[j], v s n_{j}[j]\right)$

\}$/ *$ otherwise, target $\neq \mathrm{Y} \bigwedge\left(\right.$ state $=$ talking $\bigvee$ priority $\left.\prec\left\langle j, v s n_{j}\right\rangle\right)$; the reply is deferred. *I

if target $=\mathrm{Y} \wedge$ state $=$ talking then

$/^{*} p_{i}$ need not re-send a reply to the request on exiting the meeting room * /

friend_requests $:=$ friend_requests $\cup\left\{\operatorname{Req}\left(\left\langle j, v s n_{j}\right\rangle, \mathrm{Y}\right)\right\}$;

\}$/ *$ else the request is out-of-date $* /$

$\square$ receive $A c k\left(j, s n, v f_{j}\right) \longrightarrow$

$V F_{i}:=\operatorname{merge}\left(V F_{i}, v f_{j}\right) ; / *$ adjust $V F_{i} * /$

$/ *$ let priority be $\left\langle i\right.$, vsn $\left._{i}\right\rangle *$ /

if $v s n_{i}[i]=s n \bigwedge$ state $\neq$ talking then $\left\{I^{*}\right.$ otherwise the acknowledgment is out-of-date $* /$

if $\exists A c k\left(j, s n^{\prime}, v f_{j}^{\prime}\right) \in a c k s \_s e t$ then /* remove $p_{j}$ 's previous reply */ acks_set $:=a c k s \_s e t-\left\{A c k\left(j, s n^{\prime}, v f_{j}^{\prime}\right)\right\}$;

acks_set $:=$ acks_set $\cup\left\{A c k\left(j, s n, v f_{j}\right)\right\}$;

for each $A c k\left(k, s n^{\prime \prime}, v f_{k}\right) \in$ acks_set do /* remove out-of-date acknowledgments */ if $v f_{k}[k]<V F_{i}[k]$ then $a c k s_{-} s e t:=a c k s \_s e t-\left\{A c k\left(k, s n^{\prime \prime}, v f_{k}\right)\right\}$;

if $\mid$ acks_set $\mid=N-1$ then $\{/ *$ received acknowledgments from every other philosopher $* /$ state $:=$ talking; $/ *$ enter the meeting room as a captain $* /$

$V F_{i}[i]:=V F_{i}[i]+1$;

is_captain $:=$ true;

acks_set $:=\emptyset$;

for each $\operatorname{Req}\left(\left\langle l, v s n_{l}\right\rangle, \mathrm{Y}\right) \in$ requests_set do

if target $=\mathrm{Y} \wedge V F_{i}[l]<v s n_{l}[l]$ then \{

$/ *$ capture the congenial philosopher $* /$

send $\operatorname{Start}\left(\right.$ priority, vsn $\left._{l}[l]\right)$ to $p_{l}$;

$\left.V F_{i}[l]:=\max \left(V F_{i}[l], v_{s n_{l}}[l]\right) ;\right\}$

else if $\left\langle l, v s n_{l}\right\rangle \prec$ priority then $/ *$ the request is out-of-date $* /$

requests_set $:=$ requests_set $-\left\{\operatorname{Req}\left(\left\langle l, v s n_{l}\right\rangle, \mathrm{Y}\right)\right\}$;

\} / end of if-then statement in line C.10 */

\}$/ *$ end of if-then statement in line C.4*/

$\square$ receive $\operatorname{Start}\left(\left\langle j, v s n_{j}\right\rangle, s n\right) \longrightarrow$

$/ *$ let priority be $\left\langle i, v_{s n_{i}}\right\rangle *$ /

if $v_{s n_{i}}[i]=$ sn $\bigwedge$ state $\neq$ talking then $\left\{/ *\right.$ captured by $p_{i} * /$

state $:=$ talking; $/ *$ enter the meeting room as a successor $* /$

$V F_{i}[i]:=V F_{i}[i]+1$;

acks_set $:=\emptyset$;

for each $\operatorname{Req}\left(\left\langle l, v s n_{l}\right\rangle, \mathrm{Y}\right) \in$ requests_set do $/ *$ remove the captain's request and the requests that bear a priority higher than that of the captain */

if $\left\langle l, v s n_{l}\right\rangle \preceq\left\langle j, v s n_{j}\right\rangle$ then requests_set $:=$ requests_set $-\left\{\operatorname{Req}\left(\left\langle l, v s n_{l}\right\rangle, \mathrm{Y}\right)\right\}$;

\}$/ *$ else, out-of-date message */

Fig. 6. Algorithm RA2 executed by philosopher $p_{i}$ 
E.1 $\square$ exit a forum of target $\longrightarrow$

E.2 for each $\operatorname{Req}\left(\left\langle j, v s n_{j}\right\rangle, \mathrm{Y}\right) \in$ requests_set do

E.3 if $V F_{i}[j]<v s n_{j}[j] \bigwedge \operatorname{Req}\left(\left\langle j, v s n_{j}\right\rangle, \mathrm{Y}\right) \notin$ friend_requests then

E.4 send $\operatorname{Ack}\left(i, v s n_{j}[j], V F_{i}\right)$ to $p_{j}$;

E.5 else if $V F_{i}[j] \geq v s n_{j}[j]$ then /* the request is out-of-date */

E.6 requests_set $:=$ requests_set $-\left\{\operatorname{Req}\left(\left\langle j, v s n_{j}\right\rangle, \mathrm{Y}\right)\right\}$;

E.7 $\quad$ target $:=\perp$;

E.8 friend_requests $:=\emptyset$;

E.9 priority $:=\langle i,[\infty, \ldots, \infty]\rangle$;

E.10 is_captain $:=$ false;

E.11 state $:=$ thinking;

E.12 ]

\section{Variables:}

- state: the state of $p_{i}$ (see Fig. 3 for the state transition diagram). The initial state is thinking.

- $V S N_{i}$ : a vector of length $N$, where $V S N_{i}[j]$, initialized to 0 , represents a count of requests that are made by $p_{j}$ and that are known at $p_{i}$.

- $V F_{i}$ : a vector of length $N$, where $V F_{i}[j]$, initialized to 0 , represents a count of entries to the meeting room that are made by or authorized to $p_{j}$ and that are known at $p_{i}$.

- priority: the priority of $p_{i}$. It is initialized to a minimal value $\langle i,[\infty, \ldots, \infty]\rangle$.

- target: the forum $p_{i}$ wishes to attend, or $\perp$ otherwise. It is initialized to $\perp$.

- is_captain: a boolean variable indicating if $p_{i}$ is a captain. It is initialized to false.

- requests_set: set of the most recent requests issued by other philosophers. It is initialized to $\emptyset$.

- friend_requests: set of requests that are for the same forum as $p_{i}$ 's request and that are received while $p_{i}$ is in the meeting room.

- acks_set: the set of acknowledgments to $p_{i}$ 's request. It is initialized to $\emptyset$

Fig. 6. Algorithm RA2 executed by philosopher $p_{i}$ (continued)

of the new $V F_{k}[j]$, and so also has its $V F_{i}[j]$ greater than $V F_{j}[j]$. As a result, care must be taken to prevent $p_{j}$ from miscounting its $V F_{j}[j]$ using the value learned from other philosophers. In the algorithm, a $\operatorname{Req}\left(\left\langle j, v s n_{j}\right\rangle, \mathrm{X}\right)$ arriving at $p_{i}$ is considered out-of-date and is removed right away if $V F_{i}[j] \geq v s n_{j}[j]$ (line B.5). Therefore, $p_{j}$ will never receive an $\operatorname{Ack}\left(i, v s n_{j}[j], v f_{i}\right)$ such that $v f_{i}[j]>V F_{j}[j]$.

\subsection{Analysis of RA2}

We now prove the correctness of RA2. For the purpose of analysis, we formalize the terms we used in the algorithm. Recall from the algorithm that for a philosopher $p_{i}$ to attend a forum $\mathrm{X}, p_{i}$ has to issue a request $\operatorname{Req}\left(\left\langle i, v s n_{i}\right\rangle, \mathrm{X}\right)$, and waits until one of the following two conditions is satisfied:

1. it has received an "up-to-date" acknowledgment from every other philosopher (line C.10);

2. it has received a Start from some $p_{j}$ (line D.3).

In the first case, $p_{i}$ enters state talking (or, more colloquially, enters the meeting room) as a captain, while in the second case $p_{i}$ enters talking as a successor. Also, in the second case, $p_{j}$ is $p_{i}$ 's captain, and $p_{j}$ captures $p_{i}$ at the time when it replies the Start message to $p_{i}$. In either case, we say that the request has been granted. The request ceases to exist after $p_{i}$ has left $\mathrm{X} . p_{i}$ is waiting for $\mathrm{X}$ in between the time it issues the request until the time the request is granted. $p_{i}$ is interested in $\mathrm{X}$ in between the time it issues the request until the time it leaves $X$.

We begin with mutual exclusion, for which we need the following two lemmas.
Lemma 4.1 Suppose that $p_{i}$ and $p_{j}$ have issued $\operatorname{Req}\left(\left\langle i, v s n_{i}\right\rangle, \mathrm{X}\right)$ and $\operatorname{Req}\left(\left\langle j, v s n_{j}\right\rangle, \mathrm{Y}\right)$, respectively. If $\left\langle i, v_{s n_{i}}\right\rangle \prec\left\langle j\right.$, vsn $\left._{j}\right\rangle$, then $p_{j}$ cannot enter state talking as a captain until $p_{i}$ has left $\mathrm{X}$.

Proof. Since $p_{i}$ 's request has a higher priority, the request must be issued before $p_{j}$ 's request arrives, and $p_{i}$ will not acknowledge $p_{j}$ 's request until $p_{i}$ has left X. Since in order for $p_{j}$ to enter state talking as a captain it must receive an acknowledgment from $p_{i}, p_{j}$ cannot enter state talking as a captain until $p_{i}$ has left $\mathrm{X}$.

Lemma 4.2 Suppose that a philosopher $p_{i}$ interested in $\mathrm{X}$ enters state talking as a captain at time $t_{1}$, and leaves the state at $t_{2}$. Then, no other philosopher interested in a different forum can enter state talking as a captain until all philosophers captured by $p_{i}$ in between $t_{1}$ and $t_{2}$ have left $\mathrm{X}$.

Proof. Let $\operatorname{Req}\left(\left\langle i, v s n_{i}\right\rangle, \mathrm{X}\right)$ be $p_{i}$ 's request. Suppose that some $p_{j}$ issuing $\operatorname{Req}\left(\left\langle j, v s n_{j}\right\rangle, \mathrm{Y}\right)$ enters state talking as a captain at time $t$ for some $t \geq t_{1}$ and $\mathrm{X} \neq \mathrm{Y}$. By Lemma 4.1, $\left\langle i, v s n_{i}\right\rangle \prec\left\langle j, v s n_{j}\right\rangle$. Let $p_{k}$ be a philosopher that is captured by $p_{i}$ in between $t_{1}$ and $t_{2}$, and let $\operatorname{Req}\left(\left\langle k, v s n_{k}\right\rangle, \mathrm{X}\right)$ be $p_{k}$ 's request.

Observe that because the priority of $p_{i}$ 's request is higher than that of $p_{j}$ 's request, $p_{j}$ cannot receive $p_{i}$ 's acknowledgment to $\operatorname{Req}\left(\left\langle j, v s n_{j}\right\rangle, \mathrm{Y}\right)$ until $p_{i}$ has left X. Let $A c k(i$, $\left.v s n_{j}[j], v f_{i}\right)$ be the acknowledgment. Since $p_{i}$ captures $p_{k}$, $v f_{i}[k] \geq v s n_{k}[k]$. So, immediately before $p_{j}$ enters state talking, $V F_{j}[k] \geq v f_{i}[k] \geq v s n_{k}[k]$ (because $p_{j}$ has updated its $V F_{j}[k]$ via the value of $v f_{i}[k]$ carried by $p_{i}$ 's acknowledgment). In order for $p_{j}$ to enter state talking as a captain the acknowledgment from $p_{k}$, say $A c k\left(k, v s n_{j}[j], v f_{k}\right)$, must be up-to-date. That is, $v f_{k}[k] \geq V F_{j}[k]$. So $v f_{k}[k] \geq v s n_{k}[k]$. 
To send out such an acknowledgment, $p_{k}$ must have left $\mathrm{X}$, for otherwise $p_{k}$ cannot increment its $V F_{k}[k]$ to $v s n_{k}[k]$. So $p_{j}$ cannot enter state talking as a captain while $p_{k}$ is still in X. $\square$

\section{Theorem 4.3 RA2 guarantees mutual exclusion.}

Proof. Suppose by contradiction that $p_{i}$ is in state talking attending $\mathrm{X}$ and, at the same time, $p_{j}$ is in state talking attending $\mathrm{Y}$, where $\mathrm{X} \neq \mathrm{Y}$. There are four cases to consider, depending on whether they enter state talking as a captain or as a successor. By Lemma 4.1, $p_{i}$ and $p_{j}$ cannot both enter the state as a captain; and by Lemma 4.2, it is not possible, either, that one of them enters state talking as a captain while the other as a successor. Moreover, since a captain can capture philosophers only when it is in state talking, by Lemma $4.2, p_{i}$ and $p_{j}$ cannot both enter state talking as a successor. Contradiction.

We now prove lockout freedom, for which we need the following lemmas.

Lemma 4.4 While $p_{i}$ is waiting for $\mathrm{X}$ and before it has left $\mathrm{X}$, every other $p_{j}$ can make at most two entries to the meeting room as a captain to attend a different forum.

Proof. To enter the meeting room as a captain, a philosopher must receive acknowledgments to its request from every other philosopher. Due to the vector sequence numbers maintained by the philosophers and the FIFO delivery in each communication link, after $p_{i}$ has issued a request $\operatorname{Req}\left(\left\langle i, v s n_{i}\right\rangle, \mathrm{X}\right)$, each $p_{j}$ can issue at most one request with a priority higher than $\left\langle i, v s n_{i}\right\rangle$ if $p_{j}$ subsequently enters the meeting room as a captain. This is because $p_{i}$ 's acknowledgment to $p_{j}$ 's request must be received by $p_{j}$ after $\operatorname{Req}\left(\left\langle i, v s n_{i}\right\rangle, \mathrm{X}\right)$ has arrived. So subsequent requests by $p_{j}$ must have a priority lower than $\left\langle i, v s n_{i}\right\rangle$. While waiting for $\mathrm{X}$ and before leaving $\mathrm{X}, p_{i}$ does not acknowledge any request for a different forum with a lower priority. So each $p_{j}$ interested in a different forum can make at most one entry to the meeting room as a captain in such a way that the request is received by $p_{i}$ after $p_{i}$ has issued $\operatorname{Req}\left(\left\langle i, v s n_{i}\right\rangle, \mathrm{X}\right)$.

Clearly, while $p_{i}$ is waiting for $\mathrm{X}, p_{j}$ can make at most one entry to the meeting room to attend a different forum in such a way that the request is received by $p_{i}$ before $p_{i}$ issues $\operatorname{Req}\left(\left\langle i, v s n_{i}\right\rangle, \mathrm{X}\right)$. Overall, after $p_{i}$ has issued $\operatorname{Req}\left(\left\langle i, v s n_{i}\right\rangle, \mathrm{X}\right)$ and before it has left $\mathrm{X}, p_{j}$ can make at most two entries to the meeting room as a captain to attend a different forum.

The above lemma implies that after $p_{i}$ has issued a request for X, at most $2(N-1)$ entries to the meeting room can "overtake" $p_{i}$ 's request in such a way that each entry is for a forum different from $X$, and the philosopher making this entry acts as a captain in the meeting room.

Lemma 4.5 Assume $j \neq i$. Then, at any time instance, either (1) $V F_{j}[i] \leq V F_{i}[i]$, or (2) $V F_{j}[i]=V F_{i}[i]+1$, $p_{i}$ has made a request $\operatorname{Req}\left(\left\langle i, v_{s n_{i}}\right\rangle, \mathrm{X}\right)$, where vsn $n_{i}[i]=V F_{i}[i]+1$, some philosopher $p_{k}$ has replied to the request with a start message, and $p_{i}$ has not yet received the message.

Proof. Recall from the algorithm that $p_{j}$ changes $V F_{j}[i]$ only when (a) it receives an $A c k\left(l, s n_{l}, v f_{l}\right)$ such that $v f_{l}[i]>$
$V F_{j}[i]$ (line C.2), or (b) it is in the meeting room and has replied to a request $\operatorname{Req}\left(\left\langle i, v s n_{i}^{\prime}\right\rangle, \mathrm{X}^{\prime}\right)$ with a start message (lines B.12 and C.19). Since $V F_{j}[i]=V F_{i}[i]=0$ initially and since $V F_{i}[i]$ is incremented only when $p_{i}$ has made an entry to the meeting room, if no philosopher has ever replied to $p_{i}$ 's requests with a start message, then $V F_{j}[i] \leq V F_{i}[i]$ all the time.

So if $V F_{j}[i]=v_{1}>V F_{i}[i]=v_{2}$ at some time $t$, then there must exist some $p_{k}$ such that $p_{k}$ is the first philosopher to set its $V F_{k}[i]$ to a value (say $v_{3}$ ) greater than $v_{2}$. Let $t^{\prime} \leq t$ be the time $p_{k}$ sets $V F_{k}[i]$ to $v_{3}$. Then, $p_{k}$ must have received a request $\operatorname{Req}\left(\left\langle i, v s n_{i}\right\rangle, \mathrm{X}\right)$ by $p_{i}$ such that $v s n_{i}[i]=v_{3}$, have replied to the request with a start message, and have set $V F_{k}[i]$ to $v_{3}$ via lines B.12 or C.19. Clearly, at this moment $p_{i}$ must have not yet made its $v_{3}$ th entry to the meeting room; otherwise, $V F_{i}[i]$ would have a value no less than $v_{3}$, contradicting the assumption that $V F_{i}[i]$ is still less than $v_{3}$ at time $t$. So $V F_{i}[i]<v_{3}$ at time $t^{\prime}$. Since $p_{i}$ has already made its $v_{3}$ th request, $V F_{i}[i] \geq v_{3}-1$ at $t^{\prime}$. So $V F_{i}[i]=v_{3}-1$ at $t^{\prime}$.

Let $t^{\prime \prime}$ be the time $p_{i}$ advances $V F_{i}[i]$ to $v_{3}$. Observe that $p_{i}$ will not issue any new request in between $t^{\prime}$ and $t^{\prime \prime}$. So for every philosopher $p_{l}, l \neq i, p_{l}$ can advance $V F_{l}[i]$ to at most $v_{3}$, regardless of how it learns of this value. Moreover, $p_{i}$ must not have received $p_{k}$ 's start message in between $t^{\prime}$ and $t^{\prime \prime}$, for, otherwise, $p_{i}$ will make its $v_{3}$ th entry to the meeting room. The fact that $V F_{i}[i]=v_{3}-1$ at $t^{\prime}$ and $t^{\prime} \leq t$ implies that $V F_{i}[i] \geq v_{3}-1$ at $t$. However, $V F_{i}[i]=v_{2}<v_{3}$ at $t$. So $V F_{i}[i]=v_{3}-1$ at $t$, and $t \leq t^{\prime \prime}$. So $V F_{j}[i]$ can be advanced to at most $v_{3}$ at $t$. Since at time $t V F_{j}[i]=v_{1}>V F_{i}[i]=v_{3}-1$, $V F_{j}[i]=v_{3}$ at $t$.

So at time $t, V F_{j}[i]=V F_{i}[i]+1, p_{i}$ has made a request $\operatorname{Req}\left(\left\langle i, v s n_{i}\right\rangle, \mathrm{X}\right)$ where $v s n_{i}[i]=V F_{i}[i]+1$, some philosopher $p_{k}$ has replied to the request with a start message, and $p_{i}$ has not yet received the message. The lemma therefore follows.

Lemma 4.6 Suppose $p_{j}$ has received a request $\operatorname{Req}\left(\left\langle i, v s n_{i}\right\rangle, \mathrm{X}\right)$ by $p_{i}$. Then, when the request is removed from $p_{j}$ 's requests_set, either (1) the request has ceased to exist, or (2) $p_{i}$ is already in the meeting room attending $\mathrm{X}$, or some philosopher $p_{k}$ has replied to the request with a start message (but the message has not yet arrived at $p_{i}$ ).

Proof. When $p_{j}$ receives a request $\operatorname{Req}\left(\left\langle i, v s n_{i}\right\rangle, \mathrm{X}\right)$, it keeps the request in requests_set until (a) $p_{j}$ receives another request from $p_{i}$ (line B.4), (b) $p_{j}$ enters the meeting room as a captain to attend a different forum, and $p_{j}$ 's priority is lower than $\left\langle i, v s n_{i}\right\rangle$ (line C.21), (c) $V F_{j}[i]$ is greater than or equal to $v s n_{i}[i]$ (lines B.17 and E.6), or (d) $p_{j}$ enters the meeting room as a successor and the priority of $p_{j}$ 's captain is lower than or equal to $\left\langle i, v s n_{i}\right\rangle$ (line D.8).

In Case (a), since messages are delivered in FIFO order, when $p_{j}$ receives another request from $p_{i}, p_{i}$ must have already attended $\mathrm{X}$ and so the request $\operatorname{Req}\left(\left\langle i, v s n_{i}\right\rangle, \mathrm{X}\right)$ has ceased to exist.

In Case (b), by Lemma 4.1, when $p_{j}$ enters the meeting room as a captain, $p_{i}$ must have attended $\mathrm{X}$ and so the request $\operatorname{Req}\left(\left\langle i, v s n_{i}\right\rangle, \mathrm{X}\right)$ has ceased to exist.

In Case (c), $V F_{i}[i]$ must be equal to $v s n_{i}[i]-1$ when $p_{i}$ makes the request $\operatorname{Req}\left(\left\langle i, v s n_{i}\right\rangle, \mathrm{X}\right)$, and remains in the same value until $p_{i}$ attends $\mathrm{X}$ (note that $p_{i}$ will never advance $V F_{i}[i]$ using a value learned from another philosopher). By 
Lemma 4.5 , when $p_{j}$ finds that $V F_{j}[i] \geq v s n_{i}[i]$, it must be the case that either $p_{i}$ 's request has ceased to exist, or some philosopher has replied to the request with a start message. So the lemma is also proven for this case.

In Case (d), let $p_{k}$ be $p_{j}$ 's captain, and let $\left\langle k, v s n_{k}\right\rangle$ be $p_{k}$ 's priority. Clearly, if $k=i$ then we are done. So assume that $i \neq k$. Since $p_{k}$ enters the meeting room as a captain, and since $p_{i}$ 's priority is higher than $p_{k}$ 's, $p_{k}$ must have already received $p_{i}$ 's request before $p_{k}$ enters the meeting room. There are two subcases to consider.

1. $p_{i}$ 's request is still in $p_{k}$ 's requests_set before $p_{k}$ enters the meeting room. Then $p_{k}$ and $p_{i}$ must be interested in the same forum. Then $p_{k}$ will also reply to $p_{i}$ 's request with a start message when $p_{k}$ enters the meeting room. So we are done.

2. $p_{i}$ 's request has already been removed from $p_{k}$ 's requests_set when $p_{k}$ enters the meeting room. Then $p_{k}$ must have removed the request because of a reason similar to one of the above four cases. In any case, it is easy to see that either $p_{i}$ has already attended $\mathbf{X}$, or some philosopher has sent $p_{i}$ a start message. So the case is also proven.

Lemma 4.7 Suppose $p_{i}$ 's request $\operatorname{Req}\left(\left\langle i, v s n_{i}\right\rangle, \mathrm{X}\right)$ has the highest priority among all existing requests. Then, $p_{i}$ will eventually enter the meeting room.

Proof. Given that every message is eventually delivered, we may assume that no message is currently in transit. So every philosopher has received $p_{i}$ 's request. Moreover, we shall assume that every philosopher still keeps the request in its requests_set. Otherwise, the lemma is proven because by Lemma $4.6 p_{i}$ must have entered, or will eventually enter, the meeting room.

When a philosopher $p_{j}$ receives $p_{i}$ 's request, either (1) it replies to the request immediately, or (2) it defers the reply until it has left a forum (lines B.7-13). In the latter case, $p_{j}$ must be interested in a forum different from $\mathrm{X}$, and either (2.1) $p_{j}$ is already in the meeting room, or (2.2) $p_{j}$ has not yet entered the meeting room, but its request has priority higher than that of $p_{i}$ 's request. In Case (2.1), $p_{j}$ will eventually reply to $p_{i}$ 's request when it exits the meeting room (lines E.2-4) because it can spend only finite time in the meeting room. Case 2.2 cannot be applied here because by the lemma assumption $p_{i}$ 's request has the highest priority in the system. So in either Case (1) or Case (2), $p_{j}$ will reply to $p_{i}$ 's request.

So assume that every $p_{j}, j \neq i$, has replied to $p_{i}$ 's request. If some of the replies is a Start, then clearly $p_{i}$ will enter the meeting room. Otherwise, all the replies are acknowledgments. If every acknowledgment $A c k\left(j, v s n_{i}[i], v f_{j}\right)$ is upto-date, i.e., $V F_{i}[j] \leq v f_{j}[j]$, then $p_{i}$ will enter the meeting room (lines C.8-10). So for the rest of the proof it remains to consider the case that some $A c k\left(j, v s n_{i}[i], v f_{j}\right)$ is out-of-date.

We argue that if $\operatorname{Ack}\left(j, v s n_{i}[i], v f_{j}\right)$ is out-of-date, i.e., $V F_{i}[j]>v f_{j}[j]$, then $p_{j}$ will send $p_{i}$ a new acknowledgment $\operatorname{Ack}\left(j, v s n_{i}[i], v f_{j}^{\prime}\right)$ with $v f_{j}^{\prime}[j]=v f_{j}[j]+1$. To see this, observe that the value of $V F_{j}[j]$ must be equal to $v f_{j}[j]$ when $p_{j}$ sent $A c k\left(j, v s n_{i}[i], v f_{j}\right)$. So when $p_{i}$ considers the acknowledgment out-of-date, either $V F_{j}[j]=v f_{j}[j]$, or $V F_{j}[j]>$ $v f_{j}[j]$. If $V F_{j}[j]=v f_{j}[j]$, by Lemma $4.5 p_{j}$ will make an entry to the meeting room. So $p_{j}$ will send a new acknowledgment $A c k\left(j, v s n_{i}[i], v f_{j}^{\prime}\right)$ with $v f_{j}^{\prime}[j]=v f_{j}[j]+1$ to $p_{i}$ after exiting the meeting room. If $V F_{j}[j]>v f_{j}[j]$, then $p_{j}$ must have made an entry to the meeting room after sending $\operatorname{Ack}\left(j, v s n_{i}[i], v f_{j}\right)$ to $p_{i}$. So $p_{j}$ will re-send $p_{i}$ a new acknowledgment $A c k\left(j, v s n_{i}[i], v f_{j}^{\prime}\right)$ with $v f_{j}^{\prime}[j]=v f_{j}[j]+1$. If $p_{j}$ 's new $A c k$ is up-to-date, then $p_{i}$ eventually enters the meeting room. Otherwise, $p_{j}$ must re-enter the meeting room. However, since $p_{i}$ 's request has the highest priority, by Lemma 4.1, no philosopher can act as a captain attending a different forum while $p_{i}$ is waiting for $\mathbf{X}$. So $p_{j}$ can only reenter the meeting room to attend $\mathrm{X}$. If $p_{j}$ re-enters the meeting room as a captain, then since $p_{j}$ still keeps $p_{i}$ 's request in its requests_set, $p_{j}$ will reply to $p_{i}$ 's request with a Start message when it enters the meeting room (lines C.15-19). If $p_{j}$ re-enters the meeting room as a successor, then there must exist some $p_{k}$ which acts as a captain in the meeting room attending $\mathrm{X}$. By our assumption, $p_{k}$ has also received $p_{i}$ 's request. If $p_{k}$ received $p_{i}$ 's request before it entered the meeting room, then $p_{k}$ must have sent a Start to $p_{i}$ when it entered the meeting room (lines C.15-19), regardless of the fact that it has already replied an $A c k$ to $p_{i}$. If $p_{k}$ received $p_{i}$ 's request after it has entered the meeting room, then $p_{k}$ must have also replied a Start to $p_{i}$ upon receiving $p_{i}$ 's request (lines B.9-12). In either case, $p_{i}$ will eventually receive $p_{k}$ 's Start message and will then attend $X$.

\section{Theorem 4.8 RA2 guarantees lockout freedom.}

Proof. From Lemma 4.4, after a philosopher $p_{i}$ has issued a request for $\mathrm{X}$, at most $2(N-1)$ entries to the meeting room can precede $p_{i}$ 's such that each of the entries is for a forum different from $X$, and the philosopher making this entry acts as a captain in the meeting room. Because of the following three facts:

- only a philosopher acting as a captain in the meeting room can capture philosophers to enter the meeting room,

- a philosopher spends only finite time in the meeting room, and

- Lemma 4.7 that the request with the highest priority will eventually be granted,

after $p_{i}$ has issued a request for $\mathrm{X}$, it will eventually enter the meeting room to attend $\mathrm{X}$.

\section{Theorem 4.9 RA2 allows concurrent occupancy.}

Proof. This follows directly from the fact that if some philosophers have issued requests for $\mathrm{X}$ and no philosopher is interested in a different forum, then all the requests for $\mathrm{X}$ will be acknowledged immediately when they are received by their destination philosophers. So the philosophers interested in X can attend the forum concurrently.

\subsection{Performance analysis}

In this section we analyze the performance of the algorithm from various perspectives. For message complexity, because some responses to a request may be caused by other requests, we measure the amortized cost.

Theorem 4.10 (Message Complexity) The amortized message cost per entry to the meeting room is at most $3(N-1)$. 
Proof. A philosopher $p_{i}$ multicasts a request $\operatorname{Req}\left(\left\langle i, v s n_{i}\right\rangle, \mathrm{X}\right)$ to every other philosopher when it wishes to attend a forum. A philosopher $p_{j}$ responds to $p_{i}$ 's request in one of the following ways:

1. It does not reply any message (because it learns from another philosopher that $p_{i}$ has been captured to attend a forum).

2. It replies to the request with exactly one message - either a Start or an Ack.

3. It replies to the request with an Ack and then a Start.

4. It replies to the request with at least two $A c k s$. There are two subcases: either $p_{j}$ 's replies are all $A c k \mathrm{~s}$, or are a sequence of $A c k s$ followed by a Start.

To simplify the proof, in Case 4 we consider only the second subcase. The first subcase can be proved similarly. Let the replies in this case be $m s g_{1}, m s g_{2}, \ldots, m s g_{l+1}$. This case happens when $p_{j}$, which has also made a request, say, $R e q_{1}$, is captured to attend a forum after replying $m s g_{1}$ to $p_{i} \cdot p_{j}$ sends $m s g_{2}$ to $p_{i}$ on exiting the forum. Then $p_{j}$ makes a second request $R e q_{2}$, is captured again to attend a forum, and sends $m s g_{3}$ to $p_{i}$ on exiting the forum; and so on until $p_{j}$ sends $m s g_{l}$ to $p_{i}$. Then $p_{j}$ makes the $l$ th request $R e q_{l}$, enters the meeting room as a captain, and sends $m s g_{l+1}$ (which is a Start) to $p_{i}$.

We can envisage each $m s g_{k}, 1<k \leq l+1$, as being caused by $R e q_{k-1}$ so that $m s g_{k}$ is counted as a "reply" by $p_{i}$ to $R e q_{k-1}$. So $p_{j}$ replies to $\operatorname{Req}\left(\left\langle i, v s n_{i}\right\rangle, \mathrm{X}\right)$ with $m s g_{1}$, while $p_{i}$ "replies" to $R e q_{k-1}$ with $m s g_{k}$. Still, $p_{i}$ may actually reply some messages to $R e q_{1}, \ldots, R e q_{l}$. Clearly, if $p_{i}$ does not reply to any of the requests with an Ack and then a Start (i.e., Case 3 above), then each request message generates at most two replies by the receiver. So the amortized cost per entry to the meeting room is at most $3(N-1)$, and so we are done with the proof. Even if $p_{i}$ does reply to one of the $l$ requests with an $A c k$ and then a Start, we can still amortize the cost to $3(N-1)$ because only one message (i.e., $\left.m s g_{1}\right)$ is counted as a reply by $p_{j}$ to $\operatorname{Req}\left(\left\langle i, v s n_{i}\right\rangle, \mathrm{X}\right)$. In the following we show that indeed $p_{i}$ can reply to at most one of these $l$ requests with an $A c k$ and then a Start, thereby establishing the theorem.

We first show that after $\operatorname{Req}\left(\left\langle i, v s n_{i}\right\rangle, \mathrm{X}\right)$ has ceased to exist, $p_{i}$ cannot make a new request (say, $R e q^{\prime}$ ), and reply a Start to $R e q_{k}$ when $R e q^{\prime}$ is granted for any $1 \leq k \leq l$. This is because $p_{i}$ must receive $p_{j}$ 's $A c k$ to $R e q^{\prime}$ before it can reply a Start to $R e q_{k}$. This Ack must be sent before $m s g_{k+1}$; otherwise, because messages are delivered in FIFO order, $p_{i}$ will receive $m s g_{k+1}$ before it receives the $A c k$. Then by $m s g_{k+1}$ $p_{i}$ will know that $R e q_{k}$ has ceased to exist and so will not reply a Start to $R e q_{k}$. On the other hand, the fact that $p_{j}$ 's $A c k$ to $R e q^{\prime}$ is sent before $m s g_{k+1}$ implies that $p_{j}$ receives $R e q^{\prime}$ before it replies $m s g_{k+1}$ to $\operatorname{Req}\left(\left\langle i, v s n_{i}\right\rangle, \mathrm{X}\right)$. By $R e q^{\prime}$ $p_{i}$ knows that $\operatorname{Req}\left(\left\langle i, v s n_{i}\right\rangle, \mathrm{X}\right)$ has ceased to exist, and so will not reply $m s g_{k+1}$ to $\operatorname{Req}\left(\left\langle i, v s n_{i}\right\rangle, \mathrm{X}\right)$; contradiction.

Next, we show that before $p_{i}$ issues $\operatorname{Req}\left(\left\langle i, v s n_{i}\right\rangle, \mathrm{X}\right), p_{i}$ cannot make a request (say, $R e q^{\prime \prime}$ ) and reply a Start to any $R e q_{k}$ when the request is granted. Clearly, because $m s g_{1}$ is sent after $p_{i}$ issues $\operatorname{Req}\left(\left\langle i, v s n_{i}\right\rangle, \mathrm{X}\right), R e q^{\prime \prime}$ must have ceased to exist when $p_{j}$ issues $R e q_{2}$ (and the following requests). So it remains to consider whether or not $p_{i}$ can reply a Start to $R e q_{1}$ after $R e q^{\prime \prime}$ is granted (and before it ceases to exist). If this is case, then $p_{j}$ must receive $R e q^{\prime \prime}$ and reply an $A c k$ to the request before it replies $m s g_{1}$ to $\operatorname{Req}\left(\left\langle i, v s n_{i}\right\rangle, \mathrm{X}\right)$. When $p_{i}$ receives $p_{j}$ 's $A c k$ and enters the meeting room as a captain, the Start message it replies to $R e q_{1}$ must arrive at $p_{j}$ before $\operatorname{Req}\left(\left\langle i, v s n_{i}\right\rangle, \mathrm{X}\right)$ does (because, again, messages are delivered in FIFO order). So $p_{j}$ must have already attended a forum for $\operatorname{Req} q_{1}$ when it receives $\operatorname{Req}\left(\left\langle i, v s n_{i}\right\rangle, \mathrm{X}\right)$. Recall that $m s g_{2}$ is sent when $p_{j}$ exits the forum. So $m s g_{1}$ is sent while $p_{j}$ is still in the forum. By the algorithm, if $p_{j}$ has replied an $A c k$ to $\operatorname{Req}\left(\left\langle i, v s n_{i}\right\rangle, \mathrm{X}\right)$ when it is in a forum, then the request will be placed in friend_requests (line B.16) and $p_{j}$ will not reply another message to the request on exiting the forum (line E.3). This then contradicts the fact that $p_{j}$ replies $m s g_{2}$ to $\operatorname{Req}\left(\left\langle i, v s n_{i}\right\rangle, \mathrm{X}\right)$ on exiting the forum.

From the above arguments we see that if $p_{i}$ replies a Start to some $R e q_{k}, 1 \leq k \leq l$, then the Start must be sent after $\operatorname{Req}\left(\left\langle i, v s n_{i}\right\rangle, \mathrm{X}\right)$ has been granted, and before the request ceases to exist. So for any subsequent request $R e q_{h}$, $k<h \leq l$, if $p_{i}$ replies to $R e q_{h}$ with a Start, then $p_{i}$ must still be in the forum requested by $\operatorname{Req}\left(\left\langle i, v s n_{i}\right\rangle, \mathrm{X}\right)$, and will not reply an $A c k$ to $R e q_{h}$ before it replies the Start. So only one message is replied by $p_{i}$ to $R e q_{h}$. So $p_{i}$ can reply to at most one of the requests $R e q_{1}, \ldots, R e q_{l}$ with an $A c k$ and then a Start. This completes the proof of the theorem.

Theorem 4.11 (Forum-Switch Complexity) After a philosopher $p_{i}$ has issued a request for $\mathrm{X}$, at most $2(N-1)$ rounds of passages can be initiated before a round of $\mathrm{X}$ is initiated in which $p_{i}$ makes a passage through $\mathrm{X}$.

Proof. By Lemma 4.2, the passages made by a captain and its successors must belong to the same round. So every round of passages must be initiated by a philosopher that attends the meeting room as a captain. By Lemma 4.4, after $p_{i}$ has issued a request for $\mathrm{X}$, at most $2(N-1)$ rounds of passages can be initiated before a round of $\mathrm{X}$ is initiated in which $p_{i}$ makes a passage through $\mathrm{X}$. The lemma therefore follows.

Lemma 4.12 Suppose a philosopher $p_{i}$ makes a passage $\alpha=$ $\left[t_{i, s}, t_{i, f}\right]$ through the meeting room, and $p_{i}$ acts as a captain in the meeting room. Suppose further that $k$ philosophers have been captured by $p_{i}$ while $p_{i}$ is in the meeting room (where each of them may be captured more than once). Let $R$ be the set of passages consisting of $\alpha$ and the passages made by the $k$ philosophers when they are captured by $p_{i}$. Then, $\operatorname{dim}(R) \leq k+1$.

Proof. Let $p_{j}$ be a philosopher that has been captured by $p_{i}$ in $\alpha$, and let $\beta=\left[t_{j, s}, t_{j, f}\right]$ be the passage made by $p_{j}$ when it is captured. Clearly, $t_{j, s}>t_{i, s}$. Moreover, if $p_{j}$ has been captured again by $p_{i}$ after it has made $\beta$, then $t_{j, f}<t_{i, f}$; that is, $\beta$ is contained in $\alpha$. So, to calculate $\operatorname{dim}(R)$ we can simply calculate $\operatorname{dim}\left(R^{\prime}\right)$, where $R^{\prime}$ consists of $\alpha$ and, for each $p_{j}$ that has been captured by $p_{i}$ in $\alpha$, the last passage $\gamma_{j}$ made by $p_{j}$ when it is captured by $p_{i}$ in $\alpha$. Because two different $\gamma_{j}$ and $\gamma_{l}$ (that are intervals) need not intersect, we have $\operatorname{dim}(R) \leq k+1$.

For the following theorem, we say that a philosopher is the pioneer of a round of passages if the philosopher initiates the first passage of the round (where the philosopher must attend a forum as a captain). 
Theorem 4.13 (Time Complexity) Suppose that a philosopher $p_{i}$ has made a request. Let $R$ be the set of passages that are initiated after $p_{i}$ has made the request, and that must be completed before $p_{i}$ can enter the meeting room. Then, $\operatorname{dim}(R) \leq(N-1)(3 N-2) / 2$.

Proof. Let $t_{1}$ be the time at which $p_{i}$ makes its request (say Request $\left(\left\langle i, v s n_{i}\right\rangle, \mathrm{X}\right)$ ), and $t_{2}$ be the time $p_{i}$ enters the meeting room to attend $\mathrm{X}$. Suppose that $R$ consists of $l$ rounds of passages $R_{1}, R_{2}, \ldots, R_{l}$. By the mutual exclusion property of the algorithm, $\operatorname{dim}(R)=\sum_{1 \leq k \leq l} \operatorname{dim}\left(R_{k}\right)$.

Clearly, of the $l$ rounds of passages, at most one of them is initiated before $t_{1}$. By Lemma 4.4, every other $p_{j}$ can establish at most two rounds of passages in between $t_{1}$ and $t_{2}$ such that $p_{i}$ must wait until the passages in the two rounds are completed before $p_{i}$ can enter the meeting room. So $l \leq 2(N-1)+1$. However, recall from the proof of Lemma 4.4 that of the 2 rounds of passages $p_{j}$ establishes, one must be initiated in the way that $p_{i}$ acknowledges $p_{j}$ 's request before $t_{1}$, while the other is initiated in the way that $p_{i}$ acknowledges $p_{j}$ 's request after $t_{1}$. So if a philosopher has initiated a passage $\alpha$ in between $t_{1}$ and $t_{2}$ such that $\alpha$ belongs to a round that is initiated before $t_{1}$, then the philosopher can establish at most one round of passages in between $t_{1}$ and $t_{2}$. So for the worst case scenario, we may assume that $l=2(N-1)$ and all the rounds $R_{1}, R_{2}, \ldots, R_{l}$ are initiated after $t_{1}$. Let $R_{1}, R_{2}, \ldots, R_{N-1}$ be the rounds that are initiated in the way that $p_{i}$ acknowledges the pioneers' requests before $t_{1}$, and $R_{N}, R_{N+1}, \ldots, R_{2(N-1)}$ are initiated in the way that $p_{i}$ acknowledges the pioneers' requests after $t_{1}$.

Consider each round $R_{k}$. Observe that if a philosopher $p_{j}$ has been captured by the pioneer of $R_{k}$ while the pioneer is in the meeting room, then $p_{j}$ 's next request must be received by $p_{i}$ after $t_{1}$, and so $p_{j}$ cannot be the pioneer of $R_{1}, R_{2}, \ldots, R_{N-1}$ after it has made the new request. However, $p_{j}$ can still be the pioneer of $R_{N}, R_{N+1}, \ldots, R_{2(N-1)}$ because $p_{j}$ 's new request may still have a priority higher than the priority of $p_{i}$ 's request Request $\left(\left\langle i, v s n_{i}\right\rangle, \mathrm{X}\right)$. This means that for each pioneer of $R_{N}, R_{N+1}, \ldots, R_{2(N-1)}$, the pioneer of the round may capture at most $N-2$ philosophers; while for each pioneer of $R_{1}, R_{2}, \ldots, R_{N-1}$, one can capture no philosopher, one captures one, one captures two, ..., and one captures $N-2$. By Lemma 4.12, therefore,

$$
\begin{aligned}
\operatorname{dim}(R) & =\sum_{1 \leq k \leq l} \operatorname{dim}\left(R_{k}\right) \\
& =(N-1)(3 N-2) / 2
\end{aligned}
$$

\subsection{Simulation results}

The simulation was conducted in a setting similar to that for RA1 (see Sect. 3.3). In the first experiment we studied how RA2 reacts to contention. We considered five different values of $m(2,3,5,10$, and 30) for both random forum choice and fixed forum choice. The results are shown in Fig. 7. From the two top charts we see that the average round size increases as the level of contention increases. The two second charts from the top show that the numbers of forum switches for $m=2,3,5$ and 10 (left chart), and for $m=2 *, 3 *, 5 *$ and $10 *$ (right chart) are all significantly smaller than that for $m=$
$30 *$, where philosophers use the meeting room in a mutually exclusive style. Likewise, the throughput ratio (shown in the two third charts from the top) also increases as the level of contention increases. All these results show that RA2 performs much better than RA1 does. The two bottom charts show the average number of messages $(\times(N-1))$ needed per request to the meeting room. Although, as analyzed in Theorem 4.10, the worst case message complexity of RA2 is $3(N-1)$, the average message complexity never exceeds $2.3(N-1)$ in the simulation. In contrast, RA1's message complexity is fixed to $2(N-1)$.

In the second experiment we studied the performance of RA2 with respect to the number of fora $m$. For each $m$, we considered five levels of contention: $100 \%$ (with $\mu_{\text {thinking }}=0$ ), $50 \%$ (with $\mu_{\text {thinking }}=250 \mathrm{~ms}$ ), $33.3 \%$ (with $\mu_{\text {thinking }}=$ $500 \mathrm{~ms}$ ), $20 \%$ (with $\mu_{\text {thinking }}=1000 \mathrm{~ms}$ ), and $5.9 \%$ (with $\left.\mu_{\text {thinking }}=4000 \mathrm{~ms}\right)$. The results are shown in Fig. 8. Compared with the results in Fig. 5, we see that when $m$ increases, RA2's performance decreases more gradually than RA1 does.

In the third experiment we studied how concurrency and delay are affected by various capturing policies, thereby studying the tradeoff between concurrency and delay under various entry policies. Recall that in RA2 only captains are allowed to capture. Capturing increases concurrency at the expense of delaying granting some requests to different fora. By disabling the capturing procedure in RA2, we can see how concurrency and delay are affected by the capturing procedure. Note that in this case RA2's entry policy reduces to RA1's.

We also modified RA2 to allow successors to capture, so as to see if RA2's concurrency can be further increased. It is clear that successors can capture philosophers only in some restricted way, or else livelocks could occur (because two philosophers may repeatedly attend the same forum via the capturing procedure, thereby blocking a third philosopher from attending a different forum). We considered two possible modifications: In the first modification, we allow a successor $p_{i}$ to capture a philosopher $p_{j}$ only if $p_{i}$ does not have a request for a different forum by a third philosopher. We shall use RA2* to denote this modification.

In the second modification, we allow capturing to go at most $k$ levels from a given captain for some constant $k$. More precisely, we call a philosopher captured by a captain firstgeneration successor. An $l$ th-generation successor attending a forum can capture a philosopher (also by sending it a Start message) requesting the same forum if $l<k$. The captured philosopher is called an $(l+1)$ th-generation successor, and is also allowed to capture if $(l+1)<k$. We shall use RA2 $(k)$ to denote this modification. It can be seen that RA2 can be easily modified to RA2 $(k)$. Since RA2 $(k)$ allows up to $k$ thgeneration successors, RA2(1) reduces to the original version of RA2 that was presented in Sect.4.1, and RA2(0) reduces to the version of RA2 that does not allow any philosopher to capture.

We measured how a philosopher's request is delayed by measuring the time a philosopher stays in state waiting per request. To see how this delay is affected globally and locally under different entry policies, we measured (1) the overall delay, which is the average delay for all philosophers, and (2) the captain's delay, which is the average delay for only captains. It is clear that in $\mathrm{RA} 2(k)$ and $\mathrm{RA} 2^{*}$, a round of forum must be initiated by a philosopher entering the forum 

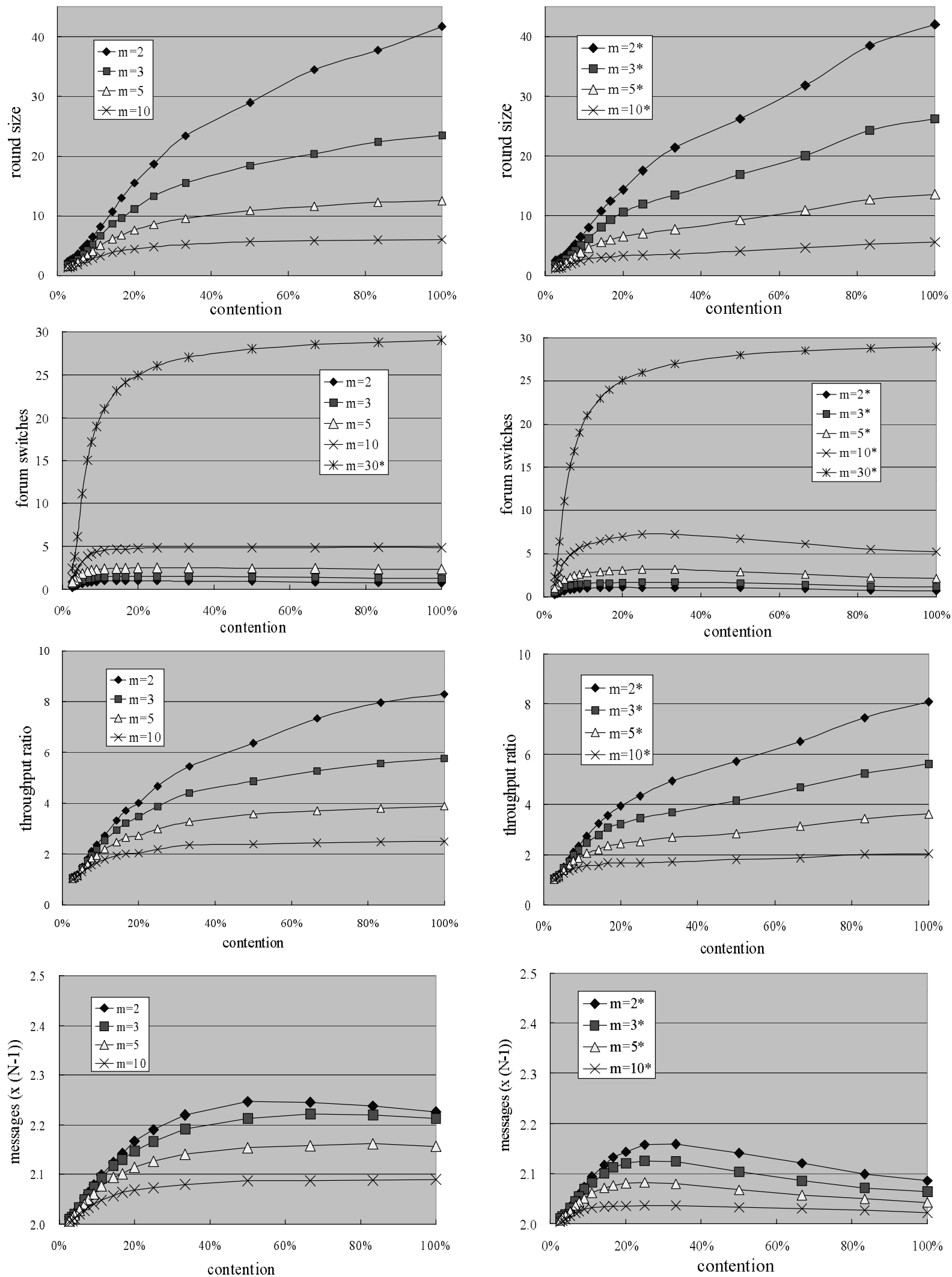

Fig. 7. RA2's performance with respect to contention. The left four charts are for random forum choice, and the right four charts are for fixed forum choice 

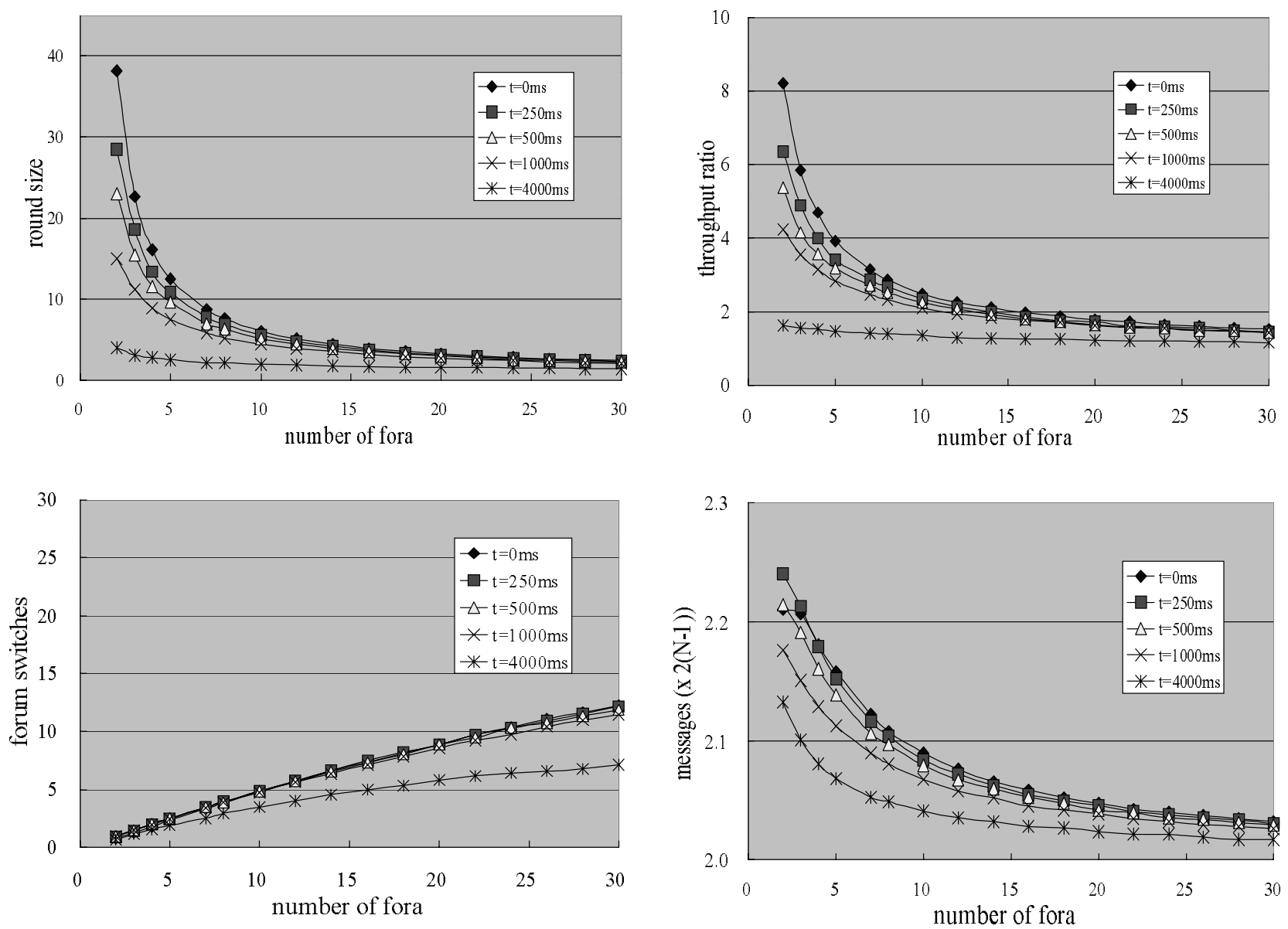

Fig. 8. RA2's performance with respect to the number of fora $m$. Time $t$ in each chart shows the value of $\mu_{\text {thinking }}$

as a captain. So the captain's delay indicates when a round of forum is ongoing and some philosophers are waiting for a different forum, how these philosophers are delayed when we allow late philosophers to jump over them to attend the ongoing forum.

In the first part of this experiment we studied the following algorithms: RA2*, RA2(0), RA2(1), RA2(3), and RA2(10) under different levels of contention. The number of fora $m$ is set to $2 *$ (with fixed forum choice). The results are shown in Fig. 9. From the results we see that capturing is crucial in boosting the performance of the system. Without capturing (as in RA2(0)), the measured values for round size, number of forum switches, throughput ratio, overall delay, and captain's delay are all significantly worse than those that allow capturing. Only message complexity is slightly reduced (to $2(N-1)$ as in RA1).

By comparing the results for RA2* and RA2(1), we see that allowing successors to capture while no other philosopher is waiting for a different forum has little help to increase RA2's concurrency. Intuitively, this is because when a philosopher $p_{i}$ requests a forum $\mathbf{X}$ while $\mathrm{X}$ is ongoing, the chances that no other philosopher is waiting for a different forum and all philosophers currently attending $X$ are successors are quite low. (If some philosopher attending $\mathrm{X}$ is a captain, $p_{i}$ will be captured by the captain as in RA2.)

Moreover, by comparing the results for RA2(1), RA2(3), and RA2(10), we see that allowing capturing to go more than one level does improve RA2's concurrency. However, the improvement from $k=1$ to $k>1$ is not as significant as the improvement from $k=0$ to $k=1$. On the other hand, captain's delays for $k=3$ and $k=10$ are generally longer than that for $k=1$ (see the bottom right chart).

In the second part of the concurrency vs. delay experiment, we then studied how $k$ affects $\operatorname{RA} 2(k)$. For each value of $k$, we consider four cases: $m=2 *$ with $\mu_{\text {thinking }}=250 \mathrm{~ms}$, $m=2 *$ with $\mu_{\text {thinking }}=1000 \mathrm{~ms}, m=5 *$ with $\mu_{\text {thinking }}=$ $250 \mathrm{~ms}$, and $m=5 *$ with $\mu_{\text {thinking }}=1000 \mathrm{~ms}$. The results are shown in Fig. 10. Again, we can see that other than message complexity, RA2(1) performs significantly better than RA2(0) does. For $k \geq 1$, from the top left chart we see that the round size increases as $k$ increases for the following three cases: $m=2 *, \mu_{\text {thinking }}=250 \mathrm{~ms}, m=2 *, \mu_{\text {thinking }}=$ $1000 \mathrm{~ms}$, and $m=5 *, \mu_{\text {thinking }}=250 \mathrm{~ms}$. Because the number of philosophers that can potentially attend the same forum is fixed (to $N / m$ ), in the above three cases the number of times a philosopher can be captured to re-enter an ongoing forum increases as the capturing level increases. Likewise, the number of forum switches (see the middle left chart) decreases as $k$ increases.

Note that the round size for $m=5 *, \mu_{\text {thinking }}=1000 \mathrm{~ms}$ is roughly unchanged for $k \geq 1$. This is because when the number of philosophers that can potentially attend the same forum is small, and the time philosophers stay in state thinking is long, there is little chance for a successor to meet some 

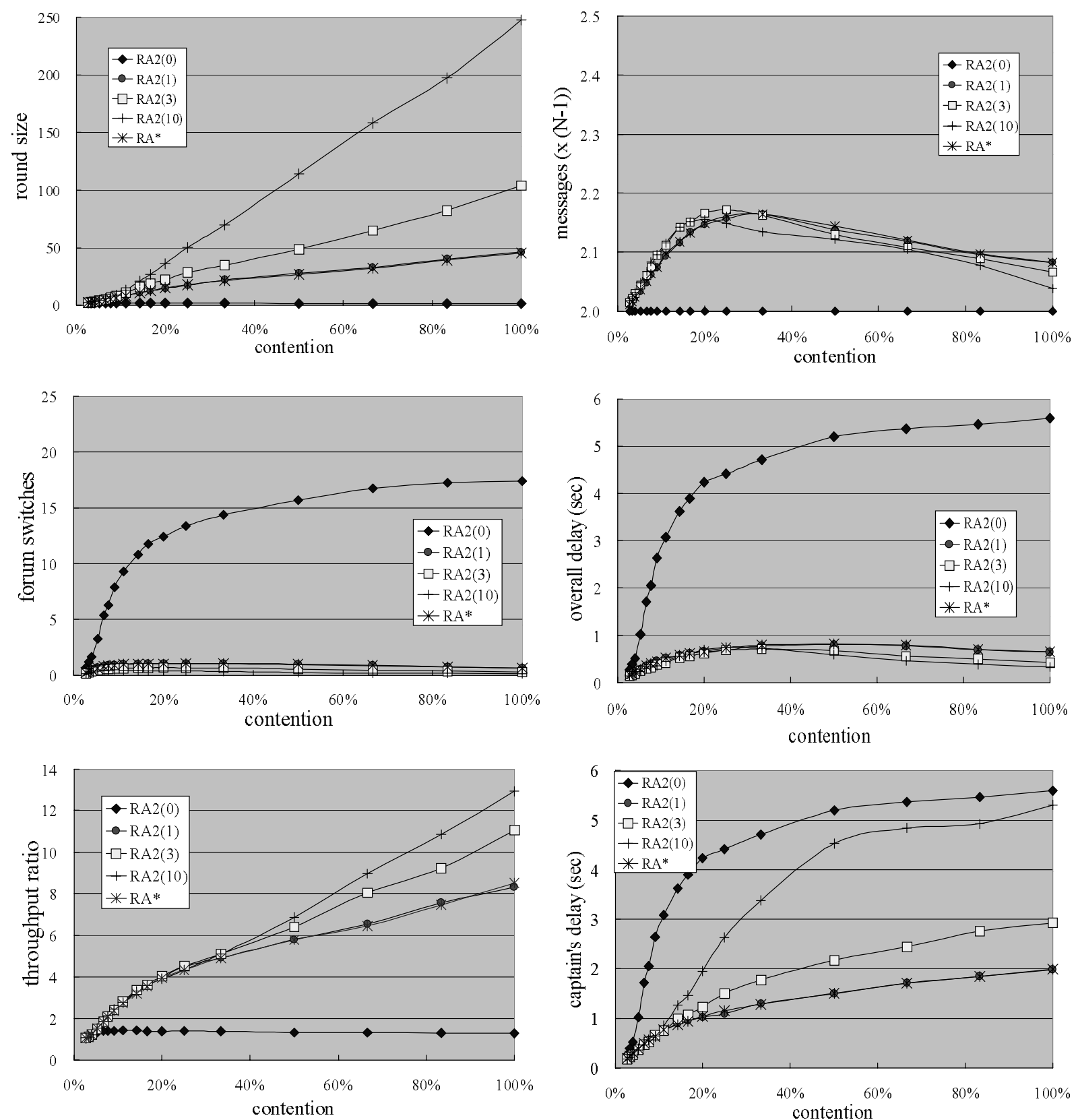

Fig. 9. Performance of RA2* and a family of RA2 $(k)$ with respect to contention. The number of fora $m$ is set to $2 *$

philosopher requesting the same forum while the successor is in a forum. Similarly, the number of forum switches is roughly not affected by $k$ for $k \geq 1$.

On the other hand, throughput ratio and overall delay stay approximately the same for all $k \geq 1$ in the four cases. This means that the overall performance of the system has almost reached its limit when $k=1$. Together with the fact that forum size increases as $k$ increases (for the cases $m=2 *$, $\mu_{\text {thinking }}=250 \mathrm{~ms} ; m=2 *, \mu_{\text {thinking }}=1000 \mathrm{~ms}$; and $m=5 *, \mu_{\text {thinking }}=250 \mathrm{~ms}$ ), we can expect that captain's delay will increase as $k$ increases. This is witnessed by the results shown in the bottom right chart.

Finally, message complexity is roughly not affected by $k$. From the results we can conclude that, in general, $k=1$ achieves the minimal delay globally and locally while offering the best throughput of the system.

\section{Conclusions and future work}

We have presented a simple algorithm RA1 for CTP. The algorithm is a straightforward modification from Ricart and Agrawala's algorithm for $n$-process mutual exclusion. We then showed that although RA1 appears to be fine from various static measures (with $2(N-1)$ in message complexity, forumswitch complexity, and time complexity), it behaves like a mutual exclusion algorithm (where only one philosopher can be in a forum at a time) when the number of fora $m$ the philosophers in CTP may like to hold increases. As analyzed in Sect. 3.2, this performance degradation is due to the combination of the following two facts:

- The entry policy that no philosopher can attend a forum if there is a higher priority request from a different philosopher waiting to attend a different forum. 

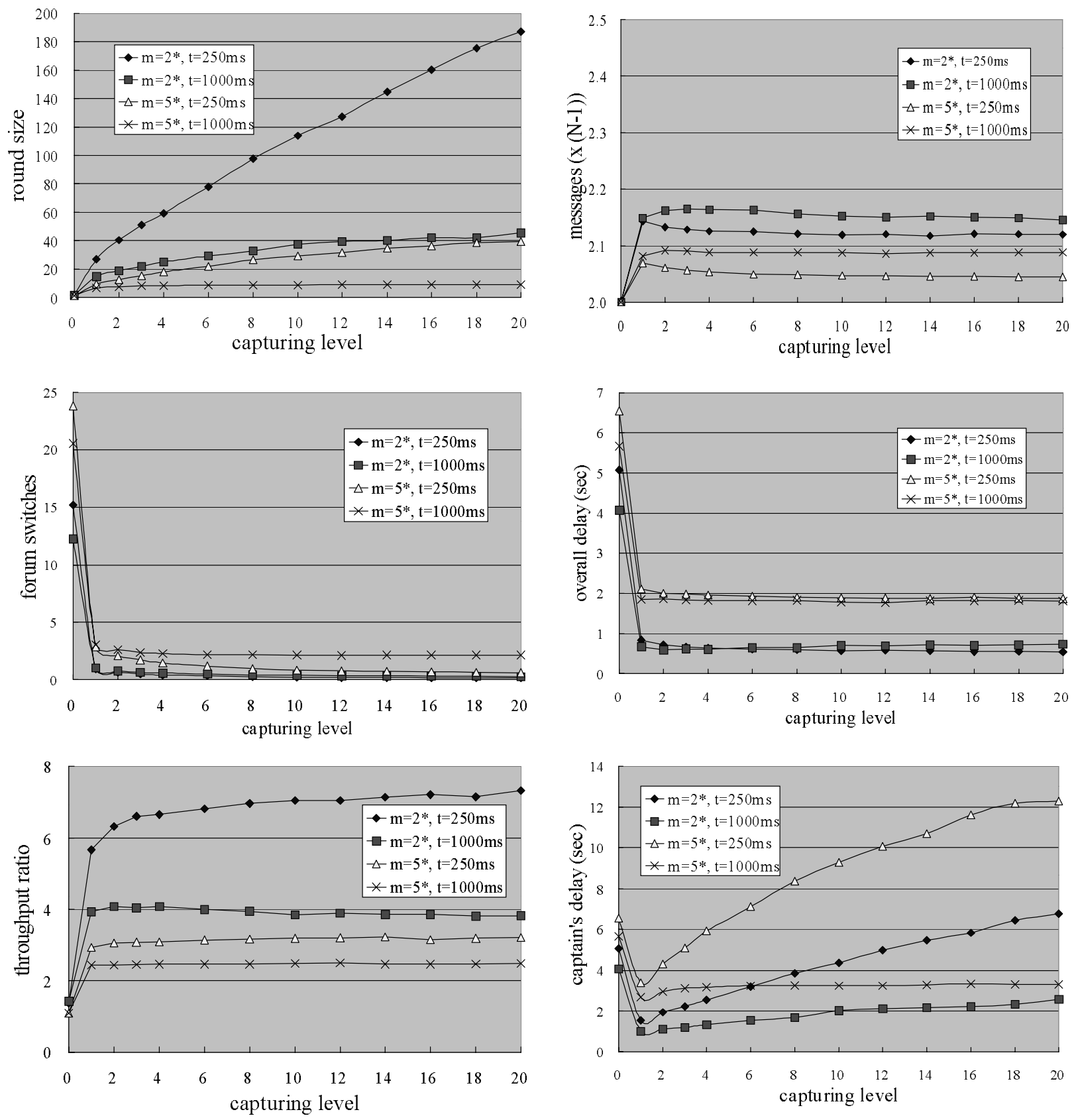

Fig. 10. Performance of RA2 $(k)$ with respect to $k$

- When two groups of philosophers request two different fora simultaneously, the "order" of their requests enforced by the system (that is used to determine the priorities of the requests) is likely to place philosophers of different groups, rather than philosophers of the same group, in an adjacent order.

Based on this finding we then proposed a weaker entry policy as follows, and presented a new algorithm RA2 to implement this policy:

- No philosopher $p_{j}$ can attend a forum $\mathrm{X}$ if there is a higher priority request from some $p_{k}$ waiting to enter a different forum, unless there is another $p_{i}$ already in $\mathrm{X}$ such that $p_{i}$ 's request has priority higher than $p_{k}$ 's request.

RA2 requires $N$ to $3(N-1)$ messages per entry to the meeting room, and has forum-switch complexity $2(N-1)$, and time complexity $O\left(N^{2}\right)$. Although these static measures do not differ much from those of RA1, RA2 performs significantly better than RA1 does at run time.

Based on RA2, we have also studied several other entry policies. Specifically, we allow capturing in RA2 to go to $k$ generations so that $l$ th-generation successors are allowed to capture if $l<k$ for some constant $k$. Our experimental results indicate that, in general, $k=1$ (i.e., RA2's original entry policy) achieves the minimal delay globally and locally while offering the best throughput of the system.

The comparison between RA1 and RA2 also highlights several directions for future work. For example, in the worst case, RA2 needs $N-1$ more messages per entry to the meet- 
ing room than RA1 does (although in the simulation RA2's average message complexity is no more than $2.3(N-1)$ ). Moreover, each request in RA2 bears a vector timestamp, while only a timestamp is needed in RA1. The use of vector timestamps compromises the scalability of RA2. Thus, it will be interesting to see if RA2 can be further improved to reduce its message complexity and message size. It will also be interesting to see, from the information structure point of view, minimum information required for exchange between processes for various entry policies.

In the paper we have chosen Ricart and Agrawala's algorithm as our first step for solving group mutual exclusion in computer networks. As we said before, the choice came from the observation that the algorithm can be straightforwardly extended to group mutual exclusion, while no other mutual exclusion algorithms we are aware of exhibit this simplicity in the extension. Nevertheless, this simple algorithm suffers a severe performance degradation and so it is interesting to see why this degradation occurs and how it can be avoided. As discussed above, the degradation is due to the fact that when two groups of processes request for critical section simultaneously, the "order" of their requests enforced by the system is likely to place processes of different groups, rather than processes of the same group, in an adjacent order. So to increase concurrency, requests cannot be granted entirely based on this order. We believe that this finding can be applied to other "permission-based" approaches to group mutual exclusion.

In the literature numerous algorithms have been proposed for mutual exclusion (see, for example, brief surveys from $[23,21,24]$ ) and for $k$-exclusion (see, e.g., $[19,25,4,3$, $13,8]$ ) in computer networks. These algorithms can be broadly classified into two categories: token based and permission based [21]. Token based algorithms achieve mutual exclusion through a unique token competed for by the processes, while in permission based algorithms a process seeks permissions to enter the critical section from some set of processes called a quorum [9]. In general, token based algorithms produce less message traffic, but have long minimum synchronization delay. (Minimum synchronization delay is the minimum delay, measured by message transmission time, from the time a process invokes a mutual exclusion request to the time it enters the critical section.) In contrast, permission based algorithms have short minimum synchronization delay, and are more fault-tolerant because a quorum usually consists of a subset of the processes in the system. Its message complexity depends on the size of the quorum a process uses. For example, the finite projective planes quorum system proposed by Maekawa [17] has quorum size $\sqrt{N}$, and so a mutual exclusion algorithm based on this quorum system requires only $O(\sqrt{N})$ messages per entry to the critical section. (For comparison, Ricart and Agrawala's algorithm needs $O(N)$ messages, and cannot tolerate any site failure.)

In light of the above discussion, it might be interesting to investigate quorum-based algorithms for group mutual exclusion. As discussed above, a process must acquire a quorum (i.e., obtains permission from every member of the quorum) in order to enter the critical section. A quorum member gives permission to only one process at a time. So a quorum system for mutual exclusion requires that every two quora in the system intersect, and a quorum system for $k$-exclusion requires that any collection of $k+1$ quora contains a pair of intersecting quora.

It is clear that that quorum systems for mutual exclusion are not suitable for group mutual exclusion, for otherwise, only one process can be in the critical section at a time. Similarly, quorum systems for $k$-exclusion cannot be applied to group mutual exclusion, for otherwise, two processes from different groups might successfully acquire two disjoint quora simultaneously and then both enter the critical section. Thus, quorum systems for group mutual exclusion must be completely redesigned. Our future work will focus on the design of quorum systems for group mutual exclusion.

Acknowledgements. The author would like to thank Kuen-Pin Wu, Wen-Jian Tsai, and Jen-Yi Liao for comments on the earlier work of this research. The author would also like to thank Vassos Hadzilacos and the anonymous referees; their comments and suggestions have substantially improved the content and the presentation of the paper. A preliminary version of this paper appeared in Proc. 13th International Symposium on DIStributed Computing (DISC99), Lecture Notes in Computer Science 1693, pp. 195-209, 1999.

\section{Appendix A: Definition on concurrent occupancy}

The definition of "concurrent occupancy" given in the paper is due to Keane and Moir [14], but the term was recently coined by Hadzilacos [10]. In the original specification of CTP [12], I used the term "concurrent entering" to exclude solutions to CTP that may impose unnecessary synchronization among philosophers attending a forum when no one else is interested in a different forum. The definition given in [12] is as follows:

Concurrent Entering: If some philosophers are interested in a forum and no philosopher is interested in a different forum, then the philosophers can attend the forum concurrently.

Unnecessary synchronization occurs, for example, in solutions that directly or indirectly apply ordinary mutual exclusion algorithms to solve the problem. The use of such algorithms forces philosophers to wait for one another in order to enter the meeting room, even if no other philosopher is interested in a different forum. However, the phrase "attend the forum concurrently" although suggestive is somewhat vague. Keane and Moir [14] later gave a different interpretation of the definition as seen in Sect. 1:

Concurrent Occupancy: If some philosopher $p$ has requested a forum $\mathrm{X}$ and no philosopher is currently attending or requesting a different forum, then $p$ can attend $X$ without waiting for other processes to leave the forum.

The definition they gave is weaker than what I intended. In particular, their definition precludes a direct application of ordinary mutual exclusion algorithms to CTP, but does not preclude the use of such algorithms as a primitive.

The distinction between these two definitions was recently clarified by Hadzilacos [10]. He called Keane and Moir's definition "concurrent occupancy", and gave a precise definition for "concurrent entering" in a shared-memory model as follows: 
If a philosopher $p$ requests a forum and no philosopher requests a different forum, then $p$ enters the meeting room within a bounded number of its own steps.

A fundamental difference between shared memory and message passing is that in shared memory, a process $p$ can communicate with another process $q$ (by reading their shared variables) without $q$ 's step, while this is not possible in message passing. Since communication is necessary in group mutual exclusion, no message-passing algorithm for CTP can satisfy Hadzilacos's definition of concurrent entering. So the paper adopted Keane and Moir's definition of "concurrent occupancy". As commented above, their definition precludes a direct application of ordinary mutual exclusion algorithms to CTP, but does not preclude the use of such algorithms as a primitive. Nevertheless, the algorithms presented in the paper do not use such primitives.

\section{References}

1. Y. Afek, D. Dolev, E. Gafni, M. Merritt, N. Shavit. A bounded first-in, first-enabled solution to the $\ell$-exclusion problem. ACM Transactions on Programming Languages and Systems, 16(3):939-953, 1994

2. K. Alagarsamy, K. Vidyasankar. Elegant solutions for group mutual exclusion problem. Technical report, Dept. of Computer Science, Memorial University of Newfoundland, St. John's, Newfoundland, Canada, 1999

3. R. Baldoni, B. Ciciani. Distributed algorithms for multiple entries to a critical section with priority. Information Processing Letters, 50(3):165-172, 1994

4. S. Bulgannawar, N.H. Vaidya. A distributed K-mutual exclusion algorithm. In Proceedings of the 15th International Conference on Distributed Computing Systems (ICDCS'95), pp. 153-160, Los Alamitos, CA, USA, May 30-June 2 1995. IEEE Computer Society Press

5. P.J. Courtois, F. Heymans, D.L. Parnas. Concurrent control with readers and writers. Communications of the ACM, 14(10):667668,1971

6. F.N. David, D.E. Barton. Combinatorial chance. London : Charles Griffin \& Co., 1962

7. M.J. Fischer, N.A. Lynch, J.E. Burns, A. Borodin. Resource allocation with immunity to limited process failure (preliminary report). In 20th Annual Symposium on Foundations of Computer Science, pp. 234-254, San Juan, Puerto Rico, 29-31 October 1979. IEEE

8. S. Fujita. A quorum based $k$-mutual exclusion by weighted $k$ quorum systems. Information Processing Letters, 67(4):191197, 1998

9. H. Garcia-Molina, D. Barbara. How to assign votes in a distributed system. Journal of the ACM, 32(4):841-860, 1985

10. V. Hadzilacos. A note on group mutual exclusion. In Proceedings of the 20th Annual ACM Symposium on Principles of Distributed Computing (PODC), Newport, Rhode Island, August 2001. ACM Press

11. C.A.R. Hoare. Communicating sequential processes. Communications of the ACM, 21(8):666-677, 1978
12. Y.-J. Joung. Asynchronous group mutual exclusion (extended abstract). In Proceedings of the 17th Annual ACM Symposium on Principles of Distributed Computing (PODC), pages 51-60, Puerto Vallarta, Mexico, June 1998. ACM Press. Full paper in Distributed Computing, 13(4):189-206, 2000

13. H. Kakugawa, S. Fujita, M. Yamashita, T. Ae. A distributed $k$-mutual exclusion algorithm using $k$-coterie. Information Processing Letters, 49(4):213-218, 1994

14. P. Keane, M. Moir. A simple local-spin group mutual exclusion algorithm. In Proceedings of the 18th Annual ACM Symposium on Principles of Distributed Computing (PODC), pp. 2332. ACM Press, 1999

15. L. Lamport. Time, clocks and the ordering of events in a distributed system. Communications of the ACM, 21(7):558-565, 1978

16. N.A. Lynch. Distributed Algorithms. Morgan-Kaufmann, 1996

17. M. Maekawa. A $\sqrt{N}$ algorithm for mutual exclusion in decentralized systems. ACM Transactions on Computer Systems, 3(2):145-159, 1985

18. F. Mattern. Virtual time and global states in distributed systems. In Proceedings of the International Workshop on Parallel and Distributed Algorithms, pp. 215-226, Gers, France, 1989. Amsterdam: North-Holland 1989

19. K. Raymond. A distributed algorithm for multiple entries to a critical section. Information Processing Letters, 30:189-193, 1989

20. M. Raynal. Algorithms for Mutual Exclusion. MIT Press, Cambridge, MA, 1986

21. M. Raynal. A simple taxonomy for distributed mutual exclusion algorithms. ACM Operating Systems Review, SIGOPS, 25(2):47-50, 1991

22. G. Ricart, A.K. Agrawala. An optimal algorithm for mutual exclusion in computer networks. Communications of the ACM, 24(1):9-17, 1981

23. B.A. Sanders. The information structure of distributed mutual exclusion algorithms. ACM Transactions on Computer Systems, 5(3):284-299, 1987

24. M. Singhal. A taxonomy of distributed mutual exclusion. Journal of Parallel and Distributed Computing, 18(1):94-101, 1993

25. P.K. Srimani, R.L.N. Reddy. Another distributed algorithm for multiple entries to a critical section. Information Processing Letters, 41(1):51-57, 1992

Yuh-Jzer Joung received his B.S. in electrical engineering from the National Taiwan University in 1984, and his M.S. and Ph.D. in computer science from the State University of New York at Stony Brook in 1988 and 1992, respectively. He was a visiting scientist at Laboratory for Computer Science, Massachusetts Institute of Technology in 1999-2000. He is currently a professor and chair in the Department of Information Management at the National Taiwan University, where he has been a faculty member since 1992 . His main research interests are in the area of distributed computing, with specific interests in process scheduling, multiparty interaction, fairness, and mutual exclusion. 